\title{
THE GROUP OF HAMILTONIAN HOMEOMORPHISMS AND CONTINUOUS HAMILTONIAN FLOWS
}

\author{
YONG-GEUN OH
}

\begin{abstract}
In this paper, we study the dynamical aspects of the Hamiltonian homeomorphism group Hameo $(M, \omega)$ which was introduced by Müller and the author. We define the notion of autonomous continuous Hamiltonian flows and extend the well-known conservation of energy to such flows. We extend the definitions of the Hofer length and of the spectral invariants $\rho_{a}$ to continuous Hamiltonian paths, and generalize the Hofer norm and the spectral norm $\gamma$ : $\operatorname{Ham}(M, \omega) \rightarrow \mathbb{R}_{+}$to the corresponding intrinsic norms on $\operatorname{Hameo}(M, \omega)$ respectively. Using this extension, we also extend Entov-Polterovich's Calabi quasi-morphism on $S^{2}$ to the space of continuous Hamiltonian paths. We also discuss a conjecture concerning extendability of Entov-Polterovich's quasimorphism and its relation to the extendability of Calabi homomorphism on the disc to $\operatorname{Hameo}\left(D^{1}, \partial D^{2}\right)$, and their implication towards the simpleness question on the area preserving homeomorphism groups of the disc $D^{2}$.
\end{abstract}

MSC2000: 53D05; 28D10.

\section{Contents}

1. Introduction

2. Hamiltonian Homeomorphism group

3. One-parameter subgroups and tangent vectors

4. Extended Hofer length and the intrinsic norm

5. Spectral invariants of continuous Hamiltonian paths

6. Calabi quasi-morphism on $\mathcal{P}_{\infty}^{\text {ham }}\left(\operatorname{Sympeo}\left(S^{2}\right), i d\right)$

7. Discussion : a wild area preserving homeomorphism on $D^{2}$

References

Date: Revision, September 2007.

Key words and phrases. hamiltonian limits, continuous Hamiltonian flows, continuous Hamiltonians, Hamiltonian homeomorphism group, area preserving homeomorphism group.

Partially supported by the NSF grant \# DMS 0503954 and a grant of the 2000 Korean Young Scientist Prize. 


\section{INTRODUCTION}

A time-dependent Hamilton's equation on a symplectic manifold $(M, \omega)$ is the first order ordinary differential equation

$$
\dot{x}=X_{H}(t, x)
$$

where the time-dependent vector field $X_{H}$ associated to a function $H: \mathbb{R} \times M \rightarrow \mathbb{R}$ is given by the defining equation

$$
\left.d H_{t}=X_{H_{t}}\right\rfloor \omega .
$$

Therefore if we consider functions $H$ that are $C^{1,1}$ so that one can apply the existence and uniqueness theorem of solutions of the above Hamilton's equation, the flow $t \mapsto \phi_{H}^{t}$, an isotopy of diffeomorphisms, is uniquely determined by the Hamiltonian $H$. We will always assume

(1) the Hamiltonians are normalized by $\int_{M} H_{t} d \mu=0$ for the Liouville measure $d \mu$ of $(M, \omega)$ if $M$ is closed,

(2) and they are compactly supported in $\operatorname{Int} M$ if $M$ is open.

We call such Hamiltonian functions normalized. For the convenience of exposition, we will focus on the closed case unless otherwise said. All the discussions in this paper equally apply for the open case too.

We denote by $C_{m}^{\infty}(M)$ the set of normalized smooth functions on $M$ and by $\mathcal{P}\left(C_{m}^{\infty}(M)\right)=C_{m}^{\infty}([0,1] \times M)$ the set of time-dependent normalized Hamiltonian functions. We will also denote the Hamiltonian isotopy generated by $H$ by

$$
\phi_{H}: t \mapsto \phi_{H}^{t}
$$

Conversely if a smooth isotopy $\lambda$ of Hamiltonian diffeomorphisms is given, we can obtain the corresponding normalized Hamiltonian $H$ by differentiating the isotopy and then solving (1.1). Therefore in the smooth category this correspondence is bijective.

On the other hand, due to the fact that this correspondence involves differentiating the function and solving Hamilton's equation, the correspondence gets murkier as the regularity of the Hamiltonian is weaker than $C^{1,1}$ because of solvability question of Hamilton's equation.

In $[\mathrm{OM}$, the author and Müller studied this relation and introduced the notion of hamiltonian limits of smooth Hamiltonian flows and proposed the notion of continuous Hamiltonian flow as the hamiltonian limits thereof. Then we introduced the $C^{0}$-concept of Hamiltonian diffeomorphisms, called Hamiltonian homeomorphisms, which forms a normal subgroup of the group of symplectic homeomorphisms : Motivated by Eliashberg's $C^{0}$-symplectic rigidity theorem [El, we defined in $\mathrm{OM}$ ] the group of symplectic homeomorphisms as follows. We give the compact-open topology on Homeo $(M)$, which is equivalent to the metric topology induced by the metric

$$
\bar{d}(\phi, \psi)=\max \left\{d_{C^{0}}(\phi, \psi), d_{C^{0}}\left(\phi^{-1}, \psi^{-1}\right)\right\}
$$

on a compact manifold $M$.

Definition 1.1 (Symplectic homeomorphism group). Define $\operatorname{Sympeo}(M, \omega)$ to be

$$
\operatorname{Sympeo}(M, \omega):=\overline{\operatorname{Symp}(M, \omega)}
$$

the $C^{0}$-closure of $\operatorname{Symp}(M, \omega)$ in $\operatorname{Homeo}(M)$ and call $\operatorname{Sympeo}(M, \omega)$ the symplectic homeomorphism group. 
We now recall the formal definition of continuous Hamiltonian flow introduced in $\mathrm{OM}$. Hofer's $L^{(1, \infty)}$ norm of Hamiltonian diffeomorphisms is defined by

$$
\|\phi\|=\inf _{H \mapsto \phi}\|H\|
$$

where $H \mapsto \phi$ means that $\phi=\phi_{H}^{1}$ is the time-one map of Hamilton's equation

$$
\dot{x}=X_{H}(t, x)
$$

and the norm $\|H\|$ is defined by

$$
\|H\|=\int_{0}^{1} \operatorname{osc} H_{t} d t=\int_{0}^{1}\left(\max _{x} H_{t}-\min _{x} H_{t}\right) d t .
$$

For two given continuous paths $\lambda, \mu:[a, b] \rightarrow H o m e o(M)$, we define their distance by

$$
\bar{d}(\lambda, \mu)=\max _{t \in[a, b]} \bar{d}(\lambda(t), \mu(t)) .
$$

Definition 1.2 (Topological Hamiltonian flow). A continuous map $\lambda: \mathbb{R} \rightarrow$ Homeo $(M)$ is called a topological Hamiltonian flow if there exists a sequence of smooth Hamiltonians $H_{i}: \mathbb{R} \times M \rightarrow \mathbb{R}$ satisfying the following :

(1) $\phi_{H_{i}} \rightarrow \lambda$ locally uniformly on $\mathbb{R} \times M$.

(2) the sequence $H_{i}$ is Cauchy in the $L^{(1, \infty)}$-topology and so has a limit $H_{\infty}$ lying in $L^{(1, \infty)}$.

We call a continuous path $\lambda:[a, b] \rightarrow H o m e o(M)$ a topological Hamiltonian path if it satisfies the same conditions with $\mathbb{R}$ replaced by $[a, b]$, and the limit $L^{(1, \infty)}$ function $H_{\infty}$ a topological Hamiltonian. In any of these cases, we say that the pair $\left(\lambda, H_{\infty}\right)$ is the hamiltonian limit of $\left(\phi_{H_{i}}, H_{i}\right)$, and write

$$
\operatorname{hlim}_{i \rightarrow \infty}\left(\phi_{H_{i}}, H_{i}\right) \rightarrow\left(\lambda, H_{\infty}\right)
$$

or sometimes even $\operatorname{hlim}_{i \rightarrow \infty}\left(\phi_{H_{i}}, H_{i}\right)=\lambda$.

We denote by $\mathcal{P}_{[a, b]}^{\text {ham }}(\operatorname{Sympeo}(M, \omega), i d)$ the set of topological Hamiltonian paths defined on $[a, b]$. When $[a, b]=[0,1]$ or when we do not specify the domain of $\lambda$, we often just write $\mathcal{P}^{\text {ham }}(\operatorname{Sympeo}(M, \omega), i d)$ for the corresponding set of topological Hamiltonian paths.

Definition 1.3 (Hamiltonian homeomorphism group). We define

$$
\operatorname{Hameo}(M, \omega)=\operatorname{ev}_{1}\left(\mathcal{P}_{[0,1]}^{\text {ham }}(\operatorname{Sympeo}(M, \omega), i d)\right.
$$

and call any element therein a Hamiltonian homeomorphisms

One basic theorem proved in $[\mathrm{OM}]$ is that $\operatorname{Hameo}(M, \omega)$ forms a normal subgroup of $\operatorname{Sympeo}(M, \omega)$ which is path-connected.

All the above discussion can be carried out using the stronger version, or $L^{\infty}$ version of Hofer's norm

$$
\|H\|_{\infty}:=\max _{t \in[0,1]}\left(\operatorname{osc} H_{t}\right) .
$$

We will call the corresponding Hamiltonian limits of smooth Hamiltonian flows continuous Hamiltonian flows and denote by

$$
\mathcal{P}_{\infty}^{\text {ham }}(\operatorname{Sympeo}(M, \omega), i d)
$$

the set of continuous Hamiltonian flows. 
The following uniqueness theorem for continuous Hamiltonian flows, which was asked in $\mathrm{OM}$ (in the $L^{(1, \infty)}$-context), was proved by Viterbo for the closed case and by the author for compactly supported case on open manifolds

Theorem 1.4 (Viterbo V2, Oh Oh9]). Suppose that $H_{i}$ is a sequence of smooth normalized Hamiltonians such that $H_{i}$ uniformly converges in $[0,1] \times M$. Then if $\phi_{H_{i}}$ converges uniformly to the constant path $i d$, then we have $\lim _{i} H_{i} \equiv 0$.

We refer to Oh9 for the locality result of $C^{0}$-Hamiltonians. The following uniqueness result in the context of $L^{(1, \infty)}$ Hamiltonians is still open.

Question 1.5. Does the uniqueness hold for $L^{(1, \infty)}$-Hamiltonians?

Because of this lack of uniqueness result in the more natural $L^{(1, \infty)}$-context, we will restrict our discussions from now on in this paper mostly to the context of continuous Hamiltonian flows. However all the results would hold true and could be proven in the same way for the topological Hamiltonian flows if this uniqueness theorem should be proved in the $L^{(1, \infty)}$ context.

Using the uniqueness result, we establish the following one-one correspondence. This extends the well-known correspondence in the smooth category to this continuous Hamiltonian category. See section 2 and 3 for more precise statements and some discussion on this correspondence in perspective.

Theorem 1.6. We have a canonical one-one correspondence

$$
\mathcal{H}_{m}^{0}([0,1] \times M, \mathbb{R}) \longleftrightarrow \mathcal{P}_{\infty}^{\text {ham }}(\operatorname{Sympeo}(M, \omega), i d)
$$

where $\mathcal{H}_{m}^{0}([0,1] \times M, \mathbb{R})$ is the set of (normalized) continuous Hamiltonians and $\mathcal{P}_{\infty}^{\text {ham }}(\operatorname{Sympeo}(M, \omega), i d)$ is the set of continuous Hamiltonian paths. And the following diagram commutes :

$$
\begin{aligned}
& C_{m}^{\infty}([0,1] \times M, \mathbb{R}) \longleftrightarrow \mathcal{P}^{h a m}(\operatorname{Symp}(M, \omega), i d) \\
& \mathcal{H}_{m}^{0}([0,1] \times M, \mathbb{R}) \longleftrightarrow \mathcal{P}_{\infty}^{\text {ham }}(\operatorname{Sympeo}(M, \omega), i d)
\end{aligned}
$$

where the vertical maps are canonical inclusion maps.

We like to point out that the set $\mathcal{H}_{m}^{0}([0,1] \times M, \mathbb{R})$ contains all $C^{1,1}$ functions and $\mathcal{P}_{\infty}^{\text {ham }}(\operatorname{Sympeo}(M, \omega), i d)$ contains all the Hamiltonian paths generated by $C^{1,1}$ functions.

The correspondence (1.4) can be interpreted as the criterion for the $C^{0}$-Hamiltonian $H$ for which weak solutions of Hamilton's equation $\dot{x}=X_{H}(t, x)$ exist. It would be interesting to make this statement more precise in the point of view of the generalized or distribution solutions of ordinary differential equations.

As we will illustrate by several theorems concerning the general properties of continuous Hamiltonian flows, this one-one correspondence will be a crucial ingredient to prove those theorems. We refer to Theorem 3.4. Theorem 3.2. Proposition 4.3. Theorem 5.3 and Theorem 6.6. for example.

It is interesting to study this correspondence between continuous Hamiltonian paths and their Hamiltonians in the point of view of Lie group theory, which is a subject of future study. However, as a step towards this goal, we prove the following theorem using the uniqueness theorem. We refer to Theorem 3.4 for a more precise statement. 
Theorem 1.7. Suppose that $\lambda \in \mathcal{P}_{\infty}^{\text {ham }}(\operatorname{Sympeo}(M, \omega), i d)$ is a one-parameter subgroup, i.e., a path satisfying

$$
\lambda(t+s)=\lambda(t) \lambda(s)
$$

whenevert, $s$ and $t+s$ lie in the domain of $\lambda$. Then its Hamiltonian $H$ is continuous and time-independent. And the converse also holds.

Similarly via the uniqueness theorem, we also prove in Theorem 3.5 that the autonomous continuous Hamiltonian flow preserves its Hamiltonian.

Theorem 1.8. Let $H$ be an autonomous continuous Hamiltonian and $\phi_{H}$ be its flow. Then we have $H \circ \phi_{H}^{s}=H$ for all $s$.

This generalizes the well-known conservation law of smooth autonomous Hamiltonian mechanics. It leads us to the following natural $C^{0}$-generalization of the existence question of a time-periodic closed orbit for autonomous Hamiltonian system

Question 1.9. Does there always exist a periodic orbit of continuous Hamiltonian flows on the hypersurface of a 'generic' level of a convex autonomous continuous Hamiltonian?

The uniqueness theorem is also essential to extend the definitions of the Hofer length and the spectral invariants constructed in $\mathrm{Oh} 5$ to continuous Hamiltonian paths : All the smooth constructions concerning the Hofer length and the spectral invariants use the Hamiltonian functions in their constructions, but not directly their associated Hamiltonian paths. They are interpreted as the invariants of the latter only via the one-one correspondence between the Hamiltonian flows and the Hamiltonian functions. Therefore extending these constructions to the topological category attached to the continuous Hamiltonian paths, not to the functions, requires this uniqueness theorem. We refer readers to sections 4 and 5 for the study of these extensions. In section 6, as an application of this generalization of spectral invariants, we extend Entov-Polterovich's study [EP] of Calabi quasi-morphisms to the space $\mathcal{P}^{\text {ham }}\left(\operatorname{Sympeo}\left(S^{2}\right), i d\right)$ of continuous Hamiltonian paths on $S^{2}$ and state a conjecture (Conjecture 6.7). Finally in section 7 we discuss an implication of this conjecture to the simpleness question of area preserving homeomorphism group of the disc.

We thank S. Müller and A. Fathi for many helpful comments and discussions.

\section{Notations}

(1) $S^{1}=\mathbb{R} / \mathbb{Z}, S^{1}(2)=\mathbb{R} / 2 \mathbb{Z}$

(2) $\pi: T^{*} N \rightarrow N$, the canonical projection of cotangent bundle.

(3) $\tilde{\pi}_{1}: T^{*}\left(S^{1}(2) \times Y\right) \rightarrow T^{*} S^{1}(2), \widetilde{\pi}_{2}: T^{*}\left(S^{1}(2) \times Y\right) \rightarrow T^{*} Y$ the obvious projections.

(4) $\pi_{1}: S^{1}(2) \times Y \rightarrow S^{1}(2), \pi_{2}: S^{1}(2) \times Y \rightarrow Y$, the obvious projections.

(5) $\iota_{Y}:[0,1] \times Y \rightarrow T^{*} Y$, the map defined by $\iota_{Y}(t, y)=o_{y}$ where $o_{y}$ the zero at $y \in T_{y}^{*} Y$. Or when $Y \subset(X, \omega)$ is a Lagrangian submanifold, $\iota_{Y}$ also denotes the corresponding map $[0,1] \times Y \rightarrow X$ induced by the inclusion.

(6) $i_{Y}: Y \rightarrow T^{*} Y, X$ the obvious inclusion maps of $Y$. 


\section{Hamiltonian HomeOMORPhism GROUP}

Following $\mathrm{OM}]$, we denote by

$$
\mathcal{P}^{\text {ham }}(\operatorname{Symp}(M, \omega), i d)
$$

the set of smooth Hamiltonian paths $\lambda:[0,1] \rightarrow \operatorname{Symp}(M, \omega)$ with $\lambda(0)=i d$, and equip it with the Hamiltonian topology (see $\mathrm{OM}$ ] or section 4 for the precise definition). It is equivalent to the metric topology induced by the metric

$$
d_{\text {ham }}(\lambda, \mu):=\bar{d}(\lambda, \mu)+\operatorname{leng}\left(\lambda^{-1} \mu\right)
$$

(Proposition $3.10 \mathrm{OM}$ ) where $\bar{d}$ is the $C^{0}$ metric on $\mathcal{P}(\operatorname{Homeo}(M), i d)$. We consider the developing map

$$
\operatorname{Dev}: \mathcal{P}^{h a m}(\operatorname{Symp}(M, \omega), i d) \rightarrow C_{m}^{\infty}([0,1] \times M, \mathbb{R}):
$$

This is defined by the assignment of the normalized generating Hamiltonian $H$ of $\lambda$, when $\lambda=\phi_{H}: t \mapsto \phi_{H}^{t}$. We also consider the inclusion map

$$
\iota_{\text {ham }}: \mathcal{P}^{\text {ham }}(\operatorname{Symp}(M, \omega), i d) \rightarrow \mathcal{P}(\operatorname{Symp}(M, \omega), i d) \hookrightarrow \mathcal{P}(\text { Homeo }(M, \omega), i d) .
$$

Imitating [OM], we call the product map $\left(\iota_{\text {ham }}\right.$, Dev) the unfolding map and denote the image thereof by

$$
\mathcal{Q}:=\operatorname{Image}\left(\iota_{\text {ham }}, \text { Dev }\right) \subset \mathcal{P}(\operatorname{Homeo}(M), i d) \times C_{m}^{0}([0,1] \times M, \mathbb{R}) .
$$

Then both maps Dev and $\iota_{h a m}$ are Lipschitz with respect to the metric $d_{h a m}$ on $\mathcal{P}^{\text {ham }}(\operatorname{Symp}(M, \omega), i d)$ by definition and so the unfolding map canonically extends to the closure $\overline{\mathcal{Q}}_{\infty}$ in $\mathcal{P}(\operatorname{Homeo}(M), i d) \times C_{m}^{0}([0,1] \times M, \mathbb{R})$ in that we have the following continuous projections

$$
\begin{aligned}
\bar{\iota}_{\text {ham }} & : \overline{\mathcal{Q}}_{\infty} \rightarrow \mathcal{P}(\text { Homeo }(M), i d) \\
\overline{\text { Dev }} & : \overline{\mathcal{Q}}_{\infty} \rightarrow C_{m}^{0}([0,1] \times M, \mathbb{R}) .
\end{aligned}
$$

We would like to note that by definition we also have the extension of the evaluation map $e v_{1}: \mathcal{P}^{\text {ham }}(\operatorname{Symp}(M, \omega), i d) \rightarrow \operatorname{Symp}(M, \omega) \rightarrow$ Homeo $(M)$ to

$$
\overline{e v}_{1}: \operatorname{Image}\left(\bar{\iota}_{\text {ham }}\right) \rightarrow \text { Homeo }(M) .
$$

The following definition was introduced in $\left[\mathrm{OM}\right.$ in the $L^{(1, \infty)}$-context but can be also applied to the present $C^{0}$-context by replacing $L^{(1, \infty)}$-norm by $L^{\infty}$-norm.

Definition 2.1 (Definition $3.18[\mathrm{OM}]$ ). We define the set $\mathcal{P}_{\infty}^{\text {ham }}(\operatorname{Sympeo}(M, \omega), i d)$ by

$$
\mathcal{P}_{\infty}^{\text {ham }}(\operatorname{Sympeo}(M, \omega), i d):=\operatorname{Image}\left(\bar{\iota}_{\text {ham }}\right) \subset \mathcal{P}(\text { Homeo }(M), i d)
$$

and call any element thereof a continuous Hamiltonian path.

In [OM], we then defined the set of $C^{0}$-Hamiltonian homeomorphisms by

$$
\operatorname{Hameo}_{\infty}(M, \omega)=\left\{h \in H \operatorname{Homeo}(M) \mid h=\overline{\operatorname{ev}}_{1}(\lambda), \lambda \in \mathcal{P}_{\infty}^{\text {ham }}(\operatorname{Sympeo}(M, \omega), i d)\right\}
$$

The following theorem was proved by Müller [Mu]

Theorem 2.2 (Müller). We have

$$
\operatorname{Hameo}_{(M, \omega)}=\operatorname{Hameo}_{\infty}(M, \omega) .
$$


Based on this, we will just write either Hamiltonian homeomorphism group by $\operatorname{Hameo}(M, \omega)$ from now on. The following normality was proved in the $L^{(1, \infty)}$ context in $\mathrm{OM}$ whose proof is rather delicate largely due to some issues related to the interplay between $L^{(1, \infty)}$-limit of Hamiltonians and $C^{0}$-limit of Hamiltonian paths. However due to Theorem 2.2 above, we can use $L^{\infty}$-limit (or equivalently $C^{0}$-limit) of smooth Hamiltonians in its proof. Then the proofs of normality and path-connectedness are very simple which we present here.

Theorem 2.3 (Theorem $1.5 \& 1.6[\mathrm{OM}]$ ). Hameo H $_{\infty}(M, \omega)$ is a path-connected normal subgroup of $\operatorname{Sympeo}(M, \omega)$ (with respect to the subspace topology).

Proof. Let $h \in$ Hameo $_{\infty}(M, \omega)$ and $g \in \operatorname{Sympeo}(M, \omega)$. By definition, there exist a sequence $H_{i}$ of Hamiltonians such that $\operatorname{hlim}_{i \rightarrow \infty}\left(\phi_{H_{i}}, H_{i}\right)=\left(h, H_{\infty}\right)$ and $\lim _{i \rightarrow \infty} \phi_{H_{i}}^{1}=h$, and a sequence $\psi_{i} \in \operatorname{Symp}(M, \omega)$ such that $\lim _{C^{0}} \psi_{i}=g$. Obviously $\lim _{C^{0}} H_{i} \circ \psi_{i}=H \circ g$ and $\psi_{i} \phi_{H_{i}} \psi_{i}^{-1}$ converges in $C^{0}$-topology and

$$
\lim _{i \rightarrow \infty} \psi_{i} \phi_{H_{i}}^{1} \psi_{i}^{-1}=g h g^{-1} \text {. }
$$

Hence by definition, we have proved $g h g^{-1} \in \operatorname{Hameo}_{\infty}(M, \omega)$ and hence the normality. Path-connectedness is immediate since the above proof shows that any element $h \in \operatorname{Hameo}_{\infty}(M, \omega)$ can be connected to the identity via a path lying in $\operatorname{Hameo}_{\infty}(M, \omega)$ that is connected in $\operatorname{Sympeo}(M, \omega)$.

In terms of this group, we also call a continuous Hamiltonian path $\lambda$ a $C^{0}$ hamiltonian-continuous map from $[0,1]$ to $\operatorname{Hameo}(M, \omega)$ and often denote it as $\lambda:[0,1] \rightarrow \operatorname{Hameo}(M, \omega)$.

Similarly to the case of the interval $[0,1]$, we can define a continuous Hamiltonian path on $[a, b]$ with $b>a$

$$
\lambda:[a, b] \rightarrow \operatorname{Hameo}(M, \omega)
$$

to be a path such that

$$
\lambda \circ(\lambda(a))^{-1} \in \mathcal{P}_{[a, b]}^{h a m}(\operatorname{Sympeo}(M, \omega), i d)
$$

where $\mathcal{P}_{\infty,[a, b]}^{\text {ham }}(\operatorname{Sympeo}(M, \omega), i d)$ is defined in the same way as $\mathcal{P}_{\infty}^{\text {ham }}(\operatorname{Sympeo}(M, \omega), i d)$ with $[0,1]$ replaced by $[a, b]$.

\section{ONE-PARAMETER SUBGROUPS AND TANGENT VECTORS}

Next, we define

$$
\mathcal{H}_{m}^{0}:=\operatorname{Image}(\overline{\mathrm{Dev}})
$$

and call any element therefrom a continuous Hamiltonain.

We first prove the following theorem by the argument used in [OM].

Theorem 3.1. The map $\overline{\operatorname{Dev}}: \overline{\mathcal{Q}}_{\infty} \rightarrow \mathcal{H}^{0}([0,1] \times M, \mathbb{R})$ is a bijective map.

Proof. Recalling that the map is nothing but the restriction to $\overline{\mathcal{Q}}_{\infty}$ of the projection

$$
\mathcal{P}(\text { Homeo }(M), i d) \times C_{m}^{0}([0,1] \times M, \mathbb{R}) \rightarrow C_{m}^{0}([0,1] \times M, \mathbb{R})
$$

and $\mathcal{H}^{0}([0,1] \times M, \mathbb{R})$ is defined to be its image of $\overline{\mathcal{Q}}_{\infty}, \overline{\text { Dev }}$ is a well-defined surjective map. To prove that it is also one-one, we need to prove that if $\left(\lambda^{\prime}, H\right),(\lambda, H) \in$ $\overline{\mathcal{Q}}_{\infty} \subset \mathcal{P}_{\infty}^{\text {ham }}(\operatorname{Sympeo}(M, \omega), i d) \times \mathcal{H}_{m}^{0}([0,1] \times M, \mathbb{R})$, then $\lambda=\lambda^{\prime}$. 
By definition, if $(\lambda, H) \in \overline{\mathcal{Q}}_{\infty}$, there exists a sequence of smooth Hamiltonians $H_{i}$ such that

$$
H_{i} \rightarrow H \quad \text { in } C^{0}, \quad \bar{d}\left(\phi_{H_{i}}, \lambda\right) \rightarrow 0 .
$$

Applying the same argument to $\left(\lambda^{\prime}, H\right)$, we obtain another sequence $H_{i}^{\prime}$ such that

$$
H_{i}^{\prime} \rightarrow H \quad \text { in } C^{0}, \quad \bar{d}\left(\phi_{H_{i}^{\prime}}, \lambda^{\prime}\right) \rightarrow 0 .
$$

Combining (3.1) and (3.2), we in particular have

$$
\left\|\bar{H}_{i} \# H_{i}^{\prime}\right\|_{\infty} \rightarrow 0 \text {. }
$$

Now we will prove the theorem by contradiction. Suppose $\lambda \neq \lambda^{\prime}$. Then, since we $\lambda(0)=\lambda(0)=i d$, there exists $s \in(0,1]$ such that $\lambda(s) \neq \lambda^{\prime}(s)$ and so $(\lambda(s))^{-1} \circ \lambda^{\prime}(s) \neq i d$. Since $(\lambda(s))^{-1} \circ \lambda^{\prime}(s)$ is a continuous map, there exists a closed symplectic ball $B \subset M$ such that

$$
(\lambda(s))^{-1} \circ \lambda^{\prime}(s)(B) \cap B=\emptyset
$$

Since $B$ is compact and $\left(\phi_{H_{i}}^{s}\right)^{-1} \circ \phi_{H_{i}^{\prime}}^{s} \rightarrow(\lambda(s))^{-1} \circ \lambda^{\prime}(s)$ uniformly, we have

$$
\left(\phi_{H_{i}}^{s}\right)^{-1} \circ \phi_{H_{i}^{\prime}}^{s}(B) \cap B=\emptyset
$$

for all sufficiently large $i$ 's. Then the energy-capacity inequality from [LM] implies

$$
\left\|\bar{H}_{i}^{s} \# H_{i}^{\prime s}\right\|_{\infty} \geq \frac{1}{2} c(B)>0
$$

where $c(B)$ is the Gromov area of the symplectic ball $B$. However we have

$$
\left\|\bar{H}_{i}^{s} \# H_{i}^{\prime s}\right\|_{\infty} \leq\left\|\bar{H}_{i} \# H_{i}^{\prime}\right\|_{\infty}
$$

which converges to 0 by (3.3). This contradicts to (3.5) and finishes the proof of $\lambda=\lambda^{\prime}$.

Combining Theorem 3.1 with the uniqueness of Hamiltonians, we immediately derive the following one-one correspondence which extends the well-known correspondence between smooth Hamiltonians and smooth Hamiltonian vector fields.

Theorem 3.2. The composition map

$$
\bar{\iota}_{\text {ham }} \circ(\overline{\mathrm{Dev}})^{-1}: \mathcal{H}_{m}^{0}([0,1] \times M, \mathbb{R}) \rightarrow \mathcal{P}_{\infty}^{\text {ham }}(\operatorname{Sympeo}(M, \omega), i d)
$$

provides a one-one correspondence between the two sets, $\mathcal{P}_{\infty}^{\text {ham }}(\operatorname{Sympeo}(M, \omega), i d)$ and $\mathcal{H}^{0}([0,1] \times M, \mathbb{R})$ under which the following diagram commutes :

$$
\begin{array}{rlc}
C^{\infty}([0,1] \times M, \mathbb{R}) & \longrightarrow & \mathcal{P}^{\text {ham }}(\operatorname{Symp}(M, \omega), i d) \\
\downarrow & & \downarrow \\
\mathcal{H}_{m}^{0}([0,1] \times M, \mathbb{R}) & \longrightarrow & \mathcal{P}_{\infty}^{\text {ham }}(\operatorname{Sympeo}(M, \omega), i d)
\end{array}
$$

The following is a natural question to ask

Question 3.3. Is $\operatorname{Hameo}(M, \omega)$ a Lie group, or does it contain a subgroup which is a Lie group bigger than $\operatorname{Ham}(M)$ ?

As a first step towards to the study of this question, we prove the following theorem

Theorem 3.4. Suppose that $\lambda \in \mathcal{P}_{\infty}^{\text {ham }}(\operatorname{Sympeo}(M, \omega), i d)$ is a continuous Hamiltonian path and $H$ its Hamiltonian. Then the followings are equivalent : 
(1) $\lambda$ is a one-parameter subgroup, i.e., a path satisfying

$$
\lambda(t+s)=\lambda(t) \lambda(s) .
$$

(2) $H$ is time-independent, i.e., there exists a continuous function $h: M \rightarrow \mathbb{R}$ such that $H=h \circ \pi(t, x)$ (i.e., $H_{t}=h$ everywhere in $\left.t \in[0,1]\right)$.

We call any such Hamiltonian an autonomous continuous Hamiltonian and denote by $\mathcal{H}_{a u t}^{0}([a, b] \times M, \mathbb{R})$ the set of autonomous Hamiltonians on $[a, b] \times M$.

Proof of Theorem 3.4. Suppose that $\lambda$ is a one-parameter subgroup. Let $H_{i}$ be a sequence of smooth Hamiltonian functions such that $\left(\phi_{H_{i}}, H_{i}\right) \rightarrow(\lambda, H)$. We consider the identity

$$
\lambda(t+s) \lambda(s)^{-1}=\lambda(t)
$$

Then for each given $s$ we have

$$
\phi_{H_{i}}^{t+s}\left(\phi_{H_{i}}^{s}\right)^{-1} \rightarrow \lambda(t+s) \lambda(s)^{-1}=\lambda(t)
$$

uniformly in $t$. We denote by $\mu_{i}^{s}=\mu_{i}^{s}(t)$ the smooth path defined by

$$
\mu_{i}^{s}(t)=\phi_{H_{i}}^{t+s}\left(\phi_{H_{i}}^{s}\right)^{-1} \text {. }
$$

Note that $\mu_{i}^{s}(0)=i d$. A straightforward computation shows

$$
\operatorname{Dev}\left(\mu_{i}^{s}\right)(t, x)=H_{i}(t+s, x)
$$

i.e., the function $G_{i}(s)$ defined by $G_{i}(s)(t, x)=H_{i}(t+s, x)$ generates the Hamiltonian flow $t \mapsto \phi_{H_{i}}^{t+s}\left(\phi_{H_{i}}^{s}\right)^{-1}$. However the latter converges to $\lambda(t+s) \lambda(s)^{-1}=\lambda(t)$ in the metric $\bar{d}$. Obviously the sequence $G_{i}(s)$ defines a Cauchy sequence in $C^{0}$ on $[c, d] \times M$ for any interval $[c, d]$. Then by the uniqueness of the Hamiltonian [V2], Oh9 we must have

$$
G_{\infty}(s)=H
$$

for all $s$ where $G_{\infty}(s)$ is the limit

$$
G_{\infty}(s):=\lim _{C^{0}} G_{i}(s) .
$$

On the other hand, using the convergence of $H_{i} \rightarrow H$ in $C^{0}$ we can also write

$$
G_{\infty}(s)(t, x)=H(t+s, x)
$$

in $C^{0}$ for each fixed $s$. Therefore combining (3.8) and (3.9), we have proved

$$
H(\cdot+s, \cdot)=H
$$

as an $C^{0}$ function for all $s$. This also implies $H_{t}=H_{s}$ for all $t, s$. Setting $h$ to be the common function, we have proved that (1) implies (2).

Conversely suppose that $H=h$ is continuous and time-independent i.e., $h(s+$ $t, x)=h(t, x)$ for all $s, t$. We need to show $\phi_{h}^{s+t}=\phi_{h}^{t} \cdot \phi_{h}^{s}$ i.e.,

$$
\phi_{h}^{s+t} \cdot\left(\phi_{h}^{s}\right)^{-1}=\phi_{h}^{t} .
$$

But the flow $t \mapsto \phi_{h}^{s+t} \cdot\left(\phi_{h}^{s}\right)^{-1}$ has been shown to be generated by the Hamiltonian $G$ defined by $G(s)(t, x)=h(t+s, x)$. But by the assumption $G(s)=h$ for all $s$.

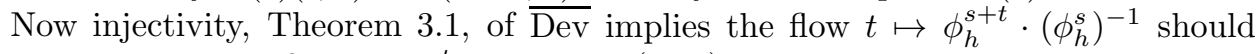
coincide with the flow $t \mapsto \phi_{h}^{t}$ and hence (3.11).

A similar argument gives rise to the law of conservation of energy under the autonomous continuous Hamiltonian flow. This extends the well-known conservation law in the smooth autonomous Hamiltonian flow. 
Theorem 3.5. Let $H$ be an autonomous continuous Hamiltonian and $\phi_{H}$ be its flow. Then we have $H \circ \phi_{H}^{s}=H$ for all $s$.

Proof. We note that the conjugate flow

$$
t \mapsto\left(\phi_{H}^{s}\right)^{-1} \phi_{H}^{t} \phi_{H}^{s}
$$

is generated by the continuous Hamiltonian $H \circ \phi_{H}^{s}$ for each $s$ : This follows by a similar argument by considering an approximating sequence $H_{i}$ and recalling that the statement holds for a smooth flow.

On the other hand, Theorem 3.4 implies that $\phi_{H}$ is a one-parameter subgroup and so we have

$$
\left(\phi_{H}^{s}\right)^{-1} \phi_{H}^{t} \phi_{H}^{s}=\phi_{H}^{t}
$$

which is generated by $H$. By the uniqueness result, we must have $H \circ \phi_{H}^{s}=H$ for all $s$. This finishes the proof.

Next, we would like to associate a vector space to each element $\phi \in \operatorname{Hameo}(M, \omega)$ which would play the role of a 'tangent vector' to $\operatorname{Hameo}(M, \omega)$ at $\phi$.

We first define the notion of 'germs' of continuous Hamiltonian paths at $\phi \in$ $\operatorname{Hameo}(M, \omega)$. For this purpose, we recall the definition of the tangent map $\overline{\operatorname{Tan}}$ : $\mathcal{P}_{\infty}^{\text {ham }}(\operatorname{Sympeo}(M, \omega), i d) \rightarrow C_{m}^{0}([0,1] \times M, \mathbb{R})$ from $[\mathrm{OM}]:$ this is defined by

$$
\operatorname{Tan}(\lambda)(t, x)=H\left(t, \phi_{H}^{t}(x)\right)
$$

if $\lambda=\phi_{H}$, i.e., if $\operatorname{Dev}(\lambda)=H$. From this, it follows that we have the identity

$$
\overline{\operatorname{Tan}}(\lambda)=\overline{\operatorname{Dev}}(\lambda) \circ \lambda .
$$

Here we note that the right hand side $\overline{\operatorname{Dev}}(\lambda) \circ \lambda$ is defined by the formula

$$
(\overline{\operatorname{Dev}}(\lambda) \circ \lambda)(t, x)=\overline{\operatorname{Dev}}(\lambda)(t, \lambda(t)(x))
$$

which we remark is well-defined as an element in $C^{0}([0,1] \times M, \mathbb{R})$ because $\overline{\operatorname{Dev}}(\lambda)$ is $C^{0}$ and $\lambda$ defines a continuous map on $[0,1] \times M$.

Definition 3.6. Let $\lambda:[a, b] \rightarrow \operatorname{Hameo}(M, \omega)$ be a continuous Hamiltonian path. We denote

$$
\lambda^{\prime}(s):=\overline{\operatorname{Tan}}(\lambda)(s)
$$

and call the hamiltonian tangent vector of the path.

By the definition of $\overline{\mathrm{Dev}}$, we also have

$$
\lambda^{\prime}(s) \circ \lambda(s)^{-1}=\overline{\operatorname{Dev}}(\lambda)(s) .
$$

Examples of hamiltonian- $C^{1}$ paths are one-parameter subgroups or continuous Hamiltonian paths with continuous Hamiltonians. For example, any piecewise smooth continuous Hamiltonian path is hamiltonian- $C^{1}$.

Now we introduce the notion of germs of continuous Hamiltonian paths.

Definition 3.7. Let $h \in \operatorname{Hameo}(M, \omega)$. Consider two hamiltonian- $C^{1}$ paths $\lambda_{1}, \lambda_{2}$ defined on $(-\varepsilon, \varepsilon)$ with $\lambda_{1}(0)=\lambda_{2}(0)=h$. We say $\lambda_{1} \sim \lambda_{2}$ at $h$ if the identity

$$
\overline{\operatorname{Dev}}\left(\lambda_{1}\right)(0)=\overline{\operatorname{Dev}}\left(\lambda_{2}\right)(0) \quad \text { in } C^{0}(M)
$$

holds.

The following lemma is an immediate consequence of the definition.

Lemma 3.8. The above relation is an equivalence relation. 
Definition 3.9 (Germs of continuous Hamiltonian paths). For a given continuous Hamiltonian path $\lambda$ issued at $h$, we denote the equivalence class of $\lambda$ at $h$ by $[\lambda]_{h}$ and call $[\lambda]_{h}$ a germ of continuous Hamiltonian paths at $h$. We denote by $\mathcal{T}_{h}$ the set of germs of the continuous Hamiltonian paths at $h$. We denote

$$
\mathcal{T}:=\bigcup_{h \in \operatorname{Hameo}(M, \omega)} \mathcal{T}_{h} .
$$

We now equip $\mathcal{T}_{h}$ with a vector space structure. For the addition, we start with the following lemma whose proof is immediate from the definitions and so omitted.

To describe $\mathcal{T}_{h}$ more concretely, let us first consider the case $h=i d$.

Lemma 3.10. (1) Let $\lambda_{1}, \lambda_{2} \in \mathcal{P}^{\text {ham }}(\operatorname{Sympeo}(M, \omega), i d)$ with $\lambda(0)=\lambda_{1}(0)=$ $\lambda_{2}(0)=i d$. Denote by $e v_{0}$ the evaluation at $t=0$, i.e., $e v_{0}(H)=H_{0}$. Let $h=e v_{0}\left(\overline{\operatorname{Dev}}\left(\lambda_{1}\right)\right)$ and $g=e v_{0}\left(\overline{\operatorname{Dev}}\left(\lambda_{2}\right)\right)$. Then $h+g$ lies in the image of $e v_{0} \circ \overline{\mathrm{Dev}}$.

(2) Let $\lambda \in \mathcal{P}^{\text {ham }}(\operatorname{Sympeo}(M, \omega), i d)$ and $k=\overline{\operatorname{Dev}}(\lambda)(0)$ and $a \in \mathbb{R}$. Then $a \cdot h$ lies in the image of $e v_{0} \circ \overline{\mathrm{Dev}}$.

Proof. We consider the product path $\lambda=\lambda_{1} \cdot \lambda_{2}$. Obviously $\lambda(0)=\lambda_{1}(0) \cdot \lambda_{2}(0)=$ $i d$. On the other hand, the identity

$$
\overline{\operatorname{Dev}}(\lambda)=\overline{\operatorname{Dev}}\left(\lambda_{1}\right)+\overline{\operatorname{Dev}}\left(\lambda_{2}\right) \circ \lambda_{1}^{-1}
$$

as an $C^{0}$-function was proved in the proof of Theorem 3.23, especially (3.28) of OM]. Recall this formula for smooth Hamiltonian paths is well-known.

We evaluate the above identity at $t=0$, which first implies that $\lambda=\lambda_{1} \cdot \lambda_{2}$ has its value given by

$$
\overline{\operatorname{Dev}}(\lambda)(0)=\overline{\operatorname{Dev}}\left(\lambda_{1}\right)(0)+\overline{\operatorname{Dev}}\left(\lambda_{2}\right)(0)=h+g .
$$

For the second statement with $a \neq 0$, we consider the rescaled path $\lambda^{a}$ defined by

$$
\lambda^{a}(t)=\lambda(a t) .
$$

Then it follows that $\overline{\operatorname{Dev}}\left(\lambda^{a}\right)(t, \cdot)=a \overline{\operatorname{Dev}}(a t, \cdot)$. This identity shows that $\lambda^{a}$ has the value given by $\overline{\operatorname{Dev}}\left(\lambda^{a}\right)(0)=a \overline{\operatorname{Dev}}(0)=a h$ which proves the statement for $a \neq 0$. When $a=0$, we just consider the constant path $\lambda \equiv i d$. This finishes the proof.

For two given continuous Hamiltonian paths $\lambda, \lambda^{\prime}$ issued at $i d$, Theorem 3.1 and the above lemma enable us to define the sum

$$
\left[\lambda_{1}\right]_{i d}+\left[\lambda_{2}\right]_{i d}=\left[\lambda_{1} \cdot \lambda_{2}\right]_{i d} .
$$

For the scalar multiplication, we define

$$
a \cdot[\lambda]_{i d}=\left[\lambda^{a}\right]_{i d} .
$$

For the general continuous Hamiltonian paths $\lambda_{1}, \lambda_{2}$ at $\phi \in \operatorname{Hameo}(M, \omega)$, we note that $\phi\left(\lambda_{1} \phi^{-1}\right)\left(\lambda_{2} \phi^{-1}\right)$ is a continuous Hamiltonian path at $\phi$ and so we define

$$
\left[\lambda_{1}\right]_{\phi}+\left[\lambda_{2}\right]_{\phi}=\left[\phi\left(\lambda_{1} \phi^{-1}\right)\left(\lambda_{2} \phi^{-1}\right)\right]_{\phi} .
$$

Similarly we define

$$
a \cdot[\lambda]_{\phi}=\left[\phi\left(\lambda \phi^{-1}\right)^{a}\right]_{\phi}
$$

noting that $\phi\left(\lambda \phi^{-1}\right)^{a}$ is a continuous Hamiltonian path at $\phi$ if $\lambda$ is so.

It is straightforward from the definition to check the following. We leave its proof to the readers. 
Proposition 3.11. The set $\mathcal{T}_{\phi}$ forms a vector space. And the maps

$$
\overline{\operatorname{Tan}}_{\phi}, \overline{\operatorname{Dev}}_{\phi}: \mathcal{T}_{\phi} \rightarrow C^{0}(M)
$$

defined by

$$
\overline{\operatorname{Tan}}_{\phi}\left([\lambda]_{\phi}\right)=\overline{\operatorname{Tan}}(\lambda)(0)
$$

and

$$
\overline{\operatorname{Dev}}_{\phi}\left([\lambda]_{\phi}\right)=\overline{\operatorname{Dev}}(\lambda)(0)
$$

respectively, define injective homomorphisms. Furthermore the image of $\overline{\operatorname{Dev}}_{\phi}$ is independent of $\phi \in \operatorname{Hameo}(M, \omega)$.

Now we denote by $\operatorname{ham}_{\infty}(M, \omega)$ the subset

$$
\operatorname{ham}_{\infty}(M, \omega):=\operatorname{Im}\left(\overline{\operatorname{Dev}}_{i d}\right) \subset C^{0}(M) .
$$

The following theorem immediately follows from Lemma 3.10 and the above discussion.

Theorem 3.12. The subset $\operatorname{ham}_{\infty}(M, \omega) \subset C^{0}(M)$ is a subspace of the vector space $C^{0}(M)$.

This theorem implies that the union $\mathcal{T} \rightarrow \operatorname{Hameo}(M, \omega)$ forms a 'vector bundle' with a canonical trivialization

$$
\overline{\operatorname{Dev}}: \mathcal{T} \rightarrow \operatorname{Hameo}(M, \omega) \times \operatorname{ham}_{\infty}(M, \omega)
$$

such that the following diagram commutes :

$$
\begin{array}{clc}
T(\operatorname{Ham}(M, \omega)) & \longrightarrow & \operatorname{Ham}(M, \omega) \times C_{m}^{\infty}(M) \\
\downarrow & \downarrow & \\
\mathcal{T} & \longrightarrow & \operatorname{Hameo}(M, \omega) \times \operatorname{ham}_{\infty}(M, \omega)
\end{array}
$$

where the horizontal maps are induced by the developing map $\overline{\mathrm{Dev}}$ defined above.

Definition 3.13. We call $\mathcal{T} \rightarrow \operatorname{Hameo}(M, \omega)$ the hamiltonian tangent bundle of $\operatorname{Hameo}(M, \omega)$.

The following questions seem to be important questions to ask.

Question 3.14. (1) Do we have a 'smooth' structure on $\operatorname{Hameo}(M, \omega)$ so that $\mathcal{T}$ becomes its tangent bundle?

(2) Is $\operatorname{Hameo}(M, \omega)$ a Lie group?

(3) Can we define a Poisson bracket on $\operatorname{ham}_{\infty}(M, \omega)$ ? Or what is the maximal subspace of $\operatorname{ham}_{\infty}(M, \omega)$ on which the bracket operation is defined in a way that it extends the standard Poisson bracket on $C_{m}^{\infty}(M)$ ?

We believe that the following conjecture is true.

Conjecture 3.15. The subset $\operatorname{ham}_{\infty}(M, \omega)$ is a proper subset of $C^{0}(M)$.

We like to compare this conjecture to the following group analog proposed in $\mathrm{OM}$

Conjecture $3.16(\underline{\mathrm{OM}})$. Hameo $(M, \omega)$ is a proper subgroup of $\operatorname{Sympeo}_{0}(M, \omega)$ in general.

It was shown in $\mathrm{OM}$ that this conjecture is true whenever the mass flow homomorphism is non-trivial or there exists a symplectic diffeomorphism that has no fixed point, e.g., $T^{2 n}$. 


\section{Extended Hofer LENGTH AND THE INTRINSIC NORM}

In this section, using the uniqueness result of continuous Hamiltonians, we will extend the definition of the Hofer length function to the continuous Hamiltonian paths, and define its associated intrinsic distance function on $\operatorname{Hameo}(M, \omega)$. In this section, we will denote by $\|\cdot\|$ the $L^{(1, \infty)}$ Hofer norm.

We give the definitions of $C^{0}$-Hamiltonian topology on $\mathcal{P}^{\text {ham }}(\operatorname{Symp}(M, \omega), i d)$ and $\operatorname{Ham}(M, \omega)$ from $[\mathrm{OM}]$.

Definition 4.1 ( $C^{0}$-Hamiltonian topology). Let $(M, \omega)$ be a closed symplectic manifold.

(1) We define the $C^{0}$-Hamiltonian topology of the set $\mathcal{P}^{\text {ham }}(\operatorname{Symp}(M, \omega), i d)$ of Hamiltonian paths by the one generated by the collection of subsets

$$
\begin{aligned}
& \mathcal{U}\left(\phi_{H}, \varepsilon_{1}, \varepsilon_{2}\right):= \\
& \left\{\phi_{H^{\prime}} \in \mathcal{P}^{\text {ham }}(\operatorname{Symp}(M, \omega), i d) \mid\left\|\bar{H} \# H^{\prime}\right\|_{\infty}<\varepsilon_{1}, \bar{d}\left(\phi_{H}, \phi_{H^{\prime}}\right)<\varepsilon_{2}\right\}
\end{aligned}
$$

of $\mathcal{P}^{\text {ham }}(\operatorname{Symp}(M, \omega), i d)$ for $\varepsilon_{1}, \varepsilon_{2}>0$ and $\phi_{H} \in \mathcal{P}^{\text {ham }}(\operatorname{Symp}(M, \omega), i d)$. We denote the resulting topological space by $\mathcal{P}_{s}^{\text {ham }}(\operatorname{Symp}(M, \omega), i d)$.

(2) We define the strong Hamiltonian topology of $\operatorname{Ham}(M, \omega)$ to be the strongest topology such that the evaluation map

$$
e v_{1}: \mathcal{P}_{s}^{\text {ham }}(\operatorname{Symp}(M, \omega), i d) \rightarrow \operatorname{Ham}(M)
$$

is continuous. We denote the resulting topological space by $\mathcal{H a m}(M, \omega)$.

We will call continuous maps with respect to the Hamiltonian topology Hamiltonian continuous.

The uniqueness theorem of Hamiltonian of a continuous Hamiltonian path enables us to define the following extension of the Hofer length to continuous Hamiltonian paths.

Definition 4.2. Let $\lambda \in \mathcal{P}_{\infty}^{\text {ham }}(\operatorname{Sympeo}(M, \omega), i d)$. We define the length of a continuous Hamiltonian path

$$
\lambda: \mathcal{P}_{\infty}^{\text {ham }}(\operatorname{Sympeo}(M, \omega), i d) \rightarrow \mathbb{R}_{+}
$$

by

$$
\operatorname{leng}(\lambda):=\|\overline{\operatorname{Dev}}(\lambda)\|=\int_{0}^{1} \operatorname{osc} H_{t} d t
$$

where $\overline{\operatorname{Dev}}(\lambda)=H$. We call this the Hofer length of the continuous Hamiltonian path $\lambda$.

The uniqueness theorem implies that $\operatorname{leng}(\lambda)$ is also the same as the limit

$$
\lim _{i \rightarrow \infty} \operatorname{leng}\left(\phi_{H_{i}}\right)=\lim _{i \rightarrow \infty}\left\|H_{i}\right\|
$$

for any sequence $\left(\phi_{i}, H_{i}\right) \rightarrow(\lambda, H)$. In particular, the definition extends that of the smooth case.

Proposition 4.3. The function leng : $\mathcal{P}_{\infty}^{\text {ham }}(\operatorname{Sympeo}(M, \omega), i d) \rightarrow \mathbb{R}$ satisfies the triangle inequality

$$
\operatorname{leng}(\lambda \mu) \leq \operatorname{leng}(\lambda)+\operatorname{leng}(\mu)
$$

and is continuous. 
Proof. The triangle inequality (4.5) is an immediate consequence of (3.14), i.e.,

$$
\overline{\operatorname{Dev}}(\lambda \mu)=\overline{\operatorname{Dev}}(\lambda)+\overline{\operatorname{Dev}}(\mu) \circ \lambda^{-1} .
$$

The triangle inequality then gives rise to the inequality

$$
|\operatorname{leng}(\lambda)-\operatorname{leng}(\mu)| \leq \operatorname{leng}\left(\lambda^{-1} \mu\right)
$$

from which continuity of leng follows.

Next we consider the action of Sympeo $(M)$ on

$$
\mathcal{P}(\operatorname{Homeo}(M), i d) \times C^{0}([0,1] \times M)
$$

given by

$$
(\psi,(\lambda, H)) \mapsto\left(\psi^{-1} \lambda \psi, H \circ \psi\right)
$$

and we prove the invariance property of the length under this action. In $\mathrm{OM}$, the action (4.6) was proven to map $\mathcal{P}_{\infty}^{\text {ham }}(\operatorname{Sympeo}(M, \omega), i d)$ to itself and so induce an action thereon. We denote this action on $\mathcal{P}_{\infty}^{\text {ham }}(\operatorname{Sympeo}(M, \omega), i d)$ by

$$
\psi \cdot \lambda=\psi^{-1} \lambda \psi .
$$

Now we prove the following theorem

Theorem 4.4. The length function defined in (4.3) is invariant under the action (4.6) on $\mathcal{P}_{\infty}^{\text {ham }}(\operatorname{Sympeo}(M, \omega), i d)$.

Proof. Let $\psi \in \operatorname{Sympeo}(M, \omega)$ and $\lambda \in \mathcal{P}_{\infty}^{\text {ham }}(\operatorname{Sympeo}(M, \omega), i d)$. Then

$$
\psi \cdot \lambda={ }_{i \rightarrow \infty}\left(\psi_{i}^{-1} \phi_{H_{i}} \psi_{i}, H_{i} \circ \psi_{i}\right)
$$

for any sequence $\psi_{i} \in \operatorname{Symp}(M, \omega)$ with $\psi_{i} \rightarrow \psi$ uniformly and $\left(\phi_{H_{i}}, H_{i}\right) \rightarrow \lambda$ in $C^{0}$-Hamiltonian topology. Therefore from the definition (4.3) and convergence (4.4), we derive

$$
\operatorname{leng}(\psi \cdot \lambda)=\lim _{i \rightarrow \infty} \operatorname{leng}\left(\psi_{i}^{-1} \phi_{H_{i}} \psi_{i}\right)=\lim _{i \rightarrow \infty} \operatorname{leng}\left(\phi_{H_{i}}\right)=\operatorname{leng}(\lambda)
$$

which finishes the proof.

Next we recall the definition of Hofer displacement energy $e(A)$ : for every compact subset $A \subset M$,

$$
\begin{aligned}
e(A) & :=\inf _{H}\left\{\|H\| \mid H \in C^{\infty}([0,1] \times M, \mathbb{R}), A \cap \phi_{H}^{1}(A)=\emptyset\right\} \\
( & \left.=\inf _{\phi}\{\|\phi\| \mid \phi \in \operatorname{Ham}(M, \omega), A \cap \phi(A)=\emptyset\}\right) .
\end{aligned}
$$

We can generalize this definition to continuous Hamiltonian category : For every compact subset $A \subset M$, we define

$$
\bar{e}(A):=\inf _{\lambda}\left\{\operatorname{leng}(\lambda) \mid \lambda \in \mathcal{P}_{\infty}^{\text {ham }}(\operatorname{Sympeo}(M, \omega), i d), A \cap \lambda(1)(A)=\emptyset\right\} .
$$

Obviously we have $e(A) \geq \bar{e}(A)$. In addition, we prove

Theorem 4.5. For every compact $A \subset M$, we have

$$
e(A)=\bar{e}(A) \text {. }
$$


Proof. For the opposite inequality, let $\delta>0$. By definition of $\bar{e}(A)$, we have $\lambda \in$ $\mathcal{P}_{\infty}^{\text {ham }}(\operatorname{Sympeo}(M, \omega), i d)$ such that $\lambda(1)(A) \cap A=\emptyset$ and

$$
\operatorname{leng}(\lambda) \leq \bar{e}(A)+\frac{\delta}{2}
$$

Let $H_{i}$ be a sequence of smooth Hamiltonians with $\operatorname{hlim}\left(\phi_{H_{i}}\right)=\lambda$. Then for all sufficiently large $i$ we have

$$
\left\|H_{i}\right\| \leq \operatorname{leng}(\lambda)+\frac{\delta}{2} \leq \bar{e}(A)+\delta .
$$

On the other hand, we have

$$
e(A) \leq\left\|H_{i}\right\|
$$

since we have $\phi_{H_{i}}^{1}(A) \cap A=\emptyset$ for all sufficiently large $i$ by the $C^{0}$-convergence of $\phi_{H_{i}}^{1} \rightarrow h, h(A) \cap A=\emptyset$ and compactness of $A$. Altogether we have derived

$$
e(A) \leq \bar{e}(A)+\delta \text {. }
$$

Since $\delta>0$ is arbitrary, this implies $e(A) \leq \bar{e}(A)$. This finishes the proof.

Based on this theorem, we will just denote by $e(A)$ the Hofer displacement energy even in the continuous Hamiltonian category. The following is an analog to the well-known fact that $e$ is invariant under the action of symplectic diffeomorphisms whose proof we omit referring to the proof of the more non-trivial case of spectral displacement energy in the next section.

Corollary 4.6. Let $g \in \operatorname{Sympeo}(M, \omega)$ and $A$ be a compact subset of $M$. Then we have

$$
e(g(A))=e(A)
$$

Now we can use the Hofer-length function generalized to the set of continuous Hamiltonian paths, and define an intrinsic norm of Hofer-type on $\operatorname{Hameo}(M, \omega)$ which in turn induces a bi-invariant distance on $\operatorname{Hameo}(M, \omega)$.

Definition 4.7 (Intrinsic norm). For any $h \in \operatorname{Hameo}(M, \omega)$, we define

$$
\|h\|=\inf _{\lambda}\left\{\operatorname{leng}(\lambda) \mid \lambda \in \mathcal{P}_{\infty}^{h a m}(\operatorname{Sympeo}(M, \omega), i d), \overline{e v}_{1}(\lambda)=h\right\} .
$$

Then we define an invariant distance function

$$
d: \operatorname{Hameo}(M, \omega) \times \operatorname{Hameo}(M, \omega) \rightarrow \mathbb{R}_{+}
$$

by $d(h, k)=\left\|h^{-1} k\right\|$.

The following theorem is the continuous Hamiltonian analog to the well-known theorem on the Hofer norm on $\operatorname{Ham}(M, \omega)[\underline{\mathrm{H}}]$.

Theorem 4.8. Let $g, h \in \operatorname{Hameo}(M, \omega)$. Then the extended Hofer norm function

$$
\|\cdot\|: \operatorname{Hameo}(M, \omega) \rightarrow \mathbb{R}_{+}
$$

is continuous in the Hamiltonian topology, and satisfies the following properties:

(1) (Symmetry)

(2) (Bi-invariance)

$\|g\|=\left\|g^{-1}\right\|$

(3) (Triangle inequality)

$\|g h\|=\|h g\|$

$\|g h\| \leq\|g\|+\|h\|$

(4) (Symplectic invariance) $\left\|\psi^{-1} g \psi\right\|=\|g\|$ for any $\psi \in \operatorname{Sympeo}(M, \omega)$,

(5) (Nondegeneracy) $\quad g=i d$ if and only if $\|g\|=0$. 
Proof. The continuity is immediate from that of the length function leng in Definition 4.2 and from the definition of $\operatorname{Hameo}(M, \omega)$ in (2.5). The symmetry and bi-invariance are straightforward to check.

For the symplectic invariance, we need to prove the identity $\|g\|=\left\|\psi^{-1} g \psi\right\|$. According to Definition 4.2 (2), we have

$$
\left\|\psi^{-1} g \psi\right\| \leq \inf _{\lambda}\left\{\operatorname{leng}\left(\psi^{-1} \lambda \psi\right) \mid \lambda \in \mathcal{P}_{\infty}^{\text {ham }}(\operatorname{Sympeo}(M, \omega), i d), e v_{1}(\lambda)=g\right\}
$$

since it follows that if $\lambda \mapsto h$, then $\psi^{-1} \lambda \psi \mapsto \psi^{-1} h \psi$. By the invariance Theorem 4.4 we have length $\left(\psi^{-1} \lambda \psi\right)=\operatorname{length}(\lambda)$. Substituting this into (4.7), we have proven $\left\|\psi^{-1} g \psi\right\| \leq\|g\|$. Applying the same argument with $\psi$ replaced by $\psi^{-1}$ and $g$ replaced by $\psi^{-1} g \psi$, we have also obtained $\left\|\psi^{-1} g \psi\right\| \geq\|g\|$, which finishes the proof.

Next we prove the triangle inequality and nondegeneracy in detail. Let $\delta>0$ be given. By Definition 4.7 there exist $\lambda, \mu \in \mathcal{P}_{\infty}^{\text {ham }}(\operatorname{Sympeo}(M, \omega), i d)$ such that

$$
\overline{e v}_{1}(\lambda)=g, \quad \overline{e v}_{1}(\mu)=h
$$

and

$$
\operatorname{leng}(\lambda) \leq\|g\|+\frac{\delta}{2}, \quad \operatorname{leng}(\mu) \leq\|h\|+\frac{\delta}{2} .
$$

By definition, there exist sequences $H_{i}, F_{i}$ of smooth Hamiltonians such that

$$
\operatorname{hlim}_{i \rightarrow \infty}\left(\phi_{i}, H_{i}\right)=\lambda, \quad \operatorname{hlim}_{i \rightarrow \infty}\left(\phi_{i}, F_{i}\right)=\mu .
$$

On the other hand, since $\overline{e v}_{1}(\lambda \mu)=g h$, we have

$$
\|g h\| \leq \operatorname{leng}(\lambda \mu) \leq \operatorname{leng}(\lambda)+\operatorname{leng}(\mu) \leq\|g\|+\|h\|+\delta .
$$

Since $\delta$ is arbitrary, we have proven the triangle inequality.

Finally we prove nondegeneracy. Suppose that $i d \neq g \in \operatorname{Hameo}(M, \omega)$. Since $g \neq i d$ is a topological homeomorphism, there exists a small symplectic ball $B(u)$ such that $g(B(u)) \cap B(u)=\emptyset$. Let $\lambda \in \mathcal{P}_{\infty}^{\text {ham }}(\operatorname{Sympeo}(M, \omega), i d)$ be any given element with $\bar{e} v_{1}(\lambda)=g$. Choose a sequence $\left(\phi_{i}, H_{i}\right)$ such that $\operatorname{hlim}\left(\phi_{i}, H_{i}\right)=\lambda$. Then we have

$$
\lim _{i \rightarrow \infty}\left\|H_{i}\right\|=\operatorname{leng}(\lambda)
$$

by (4.4). and since $B(u)$ is compact and $g(B(u)) \cap B(u) \neq \emptyset$, we also have

$$
\phi_{i}(B(u)) \cap B(u)=\emptyset
$$

for all sufficiently large $i$ because $\phi_{i} \rightarrow g$ in $C^{0}$ topology. By definition of the Hofer displacement energy, (4.9) implies

$$
\left\|H_{i}\right\| \geq\left\|\phi_{i}\right\| \geq e(B(u))>0
$$

for all sufficiently large $i$. The latter positivity follows from the energy-capacity inequality proven in $[\mathrm{LM}]$.

Then (4.8) and (4.10) imply leng $(\lambda) \geq e(B(u))>0$. Since this is true for any $\lambda \in \mathcal{P}_{\infty}^{\text {ham }}(\operatorname{Sympeo}(M, \omega), i d)$ with $\overline{e v}_{1}(\lambda)=g$, this gives rise to $\|g\| \geq e(B(u))>0$ and finishes the proof of nondegeneracy.

However Stefan Müller $\mathrm{Mu}$ pointed out that the answer to the following question is open 
Question 4.9 (S. Müller). Denote by $\|\cdot\|_{\text {Ham }}$ and $\|\cdot\|_{\text {Hameo }}$ the Hofer norm on $\operatorname{Ham}(M, \omega)$ and the extended Hofer norm on $\operatorname{Hameo}(M, \omega)$. Let $\phi \in \operatorname{Ham}(M, \omega) \subset$ $\operatorname{Hameo}(M, \omega)$. Does the following identity

$$
\|\phi\|_{\text {Ham }}=\|\phi\|_{\text {Hameo }}
$$

hold in general?

This question can be shed some light on by relating it to the general construction of path metric spaces $\left(X, d_{\ell}\right)$ starting from a general metric space $(X, d)$ in the point of view of Chapter $1 \mathrm{Gr2}$, although the question is not exactly in the context of this general construction therein because of its interplay with the $C^{0}$-metric in addition.

\section{Spectral invariants of Continuous Hamiltonian paths}

In this section, we extend the definition and basic properties of the spectral invariants of Hamiltonian paths formulated in Oh5 to continuous Hamiltonian category.

For this extension, it is crucial to have the definition in the level of Hamiltonian paths, i.e., on $\mathcal{P}^{\text {ham }}(\operatorname{Symp}(M, \omega), i d)$ as formulated in Oh5, not just on the covering space of $\operatorname{Ham}(M, \omega)$. We refer to V1, Oh1, Oh2, Sc for the earlier definition of similar invariants in the context of exact cases. Furthermore the uniqueness theorem of continuous Hamiltonians will be crucial for the extension to the $C^{0}$ category.

We first recall the definition and basic properties of the spectral invariants $\rho(H ; a)$ for a time-periodic Hamiltonian $H$ from Oh5, but with some twists to incorporate the Hamiltonian topology in its presentation. We refer readers to Oh5 for the complete discussion on general properties of $\rho(H ; a)$.

For a given time-periodic Hamiltonian and a choice of time-periodic almost complex structure $J$, we consider the perturbed Cauchy-Riemann equation

$$
\frac{\partial u}{\partial \tau}+J\left(\frac{\partial u}{\partial t}-X_{H}(t, u)\right)=0
$$

and its associated Floer complex $\partial_{(H, J)}: C F(H) \rightarrow C F(H)$. We call a Floer chain $\alpha$ a Floer cycle if $\partial_{(H, J)} \alpha=0$ and denote its homology class by $[\alpha]$.

Definition 5.1 (Definition \& Theorem $7.7[\mathrm{Oh} 5]$ ). Let $H$ be time-periodic Hamiltonian. Let $a \neq 0$ be a given quantum cohomology class in $Q H^{*}(M)$, and denote by $a^{b} \in F H_{*}$ the Floer homology class dual to $a$ in the sense of [Oh5]. For any given Hamiltonian path $\lambda=\phi_{H} \in \mathcal{P}^{\text {ham }}(\operatorname{Symp}(M, \omega), i d)$ such that $H$ is non-degenerate in the Floer theoretic sense, we define

$$
\rho(\lambda ; a):=\rho(H ; a)=\inf _{\alpha \in \operatorname{ker} \partial_{H}}\left\{\lambda_{H}(\alpha) \mid[\alpha]=a^{b}\right\}
$$

where $a^{b}$ is the dual to the quantum cohomology class $a$ in the sense of Oh5. Then this number is finite for any quantum cohomology class $a \neq 0$. We call any of these spectral invariants of the Hamiltonian path $\lambda$.

Now let $H:[0,1] \times M \rightarrow \mathbb{R}$ be any smooth Hamiltonian, not necessarily periodic and let $\lambda=\phi_{H}$ be its Hamiltonian path. We now explain how we associate the spectral invariant $\rho(\lambda ; a)$ to such a path $\lambda$. 
Out of the given Hamiltonian $H$, we consider the time-periodic Hamiltonian of the type $H^{\zeta}$ where $\zeta$ is a reparameterization of $[0,1]$ of the type

$$
\zeta(t)= \begin{cases}0 & \text { for } 0 \leq t \leq \frac{\varepsilon_{0}}{2} \\ 1 & \text { for } 1-\frac{\varepsilon_{0}}{2} \leq t \leq 1\end{cases}
$$

and

$$
\zeta^{\prime}(t) \geq 0 \text { for all } t \in[0,1]
$$

and the reparameterized Hamiltonian by $H^{\zeta}$ is given by

$$
H^{\zeta}(t, x)=\zeta^{\prime}(t) H(\zeta(t), x)
$$

which generates the Hamiltonian isotopy $t \mapsto \phi_{H}^{\zeta(t)}$ in general. The following norm,

$$
\|\zeta-i d\|_{h a m}=\|\zeta-i d\|_{C^{0}}+\int_{0}^{1}\left|\zeta^{\prime}(t)-1\right| d t
$$

which measures a distance of $\zeta$ from the identity parametrization, turns out to be useful as illustrated in $\mathrm{OM}$.

To assign a well-defined number $\rho(\lambda ; a)$ depending only on $H$ itself not on its reparameterization $H^{\zeta}$, we note that any two such reparameterized Hamiltonian paths are homotopic to each other. The homotopy invariance axiom of the spectral invariants from Oh5, Oh7] imply that the following definition is well-defined in that it does not depend on the choice of $\zeta$.

Definition 5.2. Let $\lambda$ be any smooth Hamiltonian path and $H$ be its generating Hamiltonian. We pick a $\zeta$ so that $\|\zeta-i d\|_{\text {ham }}$ so small that all the properties in the $C^{0}$-approximation Lemma OM hold. Then we define

$$
\rho(\lambda ; a):=\rho\left(H^{\zeta} ; a\right) .
$$

In Oh3], Oh5], we proved the general inequality

$$
\int_{0}^{1}-\max _{x}(K-H) d t \leq \rho(K ; a)-\rho(H ; a) \leq \int_{0}^{1}-\min _{x}(K-H) d t
$$

for two nondegenerate Hamiltonian functions $H, K$. This enabled us to extend the definition of $\rho(a ; \cdot)$ to arbitrary smooth Hamiltonian $H$ by setting

$$
\rho(H ; a)=\rho\left(H^{\zeta} ; a\right) .
$$

Theorem 5.3. For a smooth Hamiltonian path $\phi_{H}$, we define

$$
\rho\left(\phi_{H} ; a\right)=\rho(H ; a) .
$$

Then the map $\rho_{a}: \phi_{H} \mapsto \rho\left(\phi_{H} ; a\right)$ extends to a continuous function

$$
\bar{\rho}_{a}=\bar{\rho}(\cdot ; a): \mathcal{P}_{\infty}^{\text {ham }}(\operatorname{Sympeo}(M, \omega), i d) \rightarrow \mathbb{R}
$$

(in the Hamiltonian topology) and satisfies the triangle inequality

$$
\bar{\rho}(\lambda \mu ; a \cdot b) \leq \bar{\rho}(\lambda ; a)+\bar{\rho}(\mu ; b) .
$$

Proof. The first statement is an immediate consequence of Hamiltonian continuity of

$$
\rho_{a}: \mathcal{P}^{h a m}(\operatorname{Symp}(M, \omega), i d) \rightarrow \mathbb{R}
$$

and the uniqueness theorem of continuous Hamiltonians from [V2], Oh9] : for any continuous Hamiltonian path $\lambda$, we define

$$
\bar{\rho}(\lambda ; a)=\lim _{i \rightarrow \infty} \rho\left(H_{i} ; a\right)
$$


for any Cauchy sequence $\left(\phi_{H_{i}}, H_{i}\right) \rightarrow \lambda$. The uniqueness theorem implies that this definition is well-defined. And then (5.4) proves continuity of the extension on $\mathcal{P}_{\infty}^{\text {ham }}(\operatorname{Sympeo}(M, \omega), i d)$.

For the proof of triangle inequality, choose any smooth sequences $\lambda_{i}$ and $\mu_{i}$ converging to $\lambda$ and $\mu$ respectively in the Hamiltonian topology. For smooth Hamiltonian paths, the inequality

$$
\rho\left(\lambda_{i} \mu_{i} ; a \cdot b\right) \leq \rho\left(\lambda_{i} ; a\right)+\rho\left(\mu_{i} ; b\right) .
$$

was proven in Oh5] (See [Sc also for the exact case). Using the continuity of $\rho$ and taking the limit of this inequality, we have proved (5.5).

Now we focus on the invariant $\rho(\lambda ; 1)$ for $1 \in Q H^{*}(M)$. Recall the function

$$
\operatorname{leng}_{\gamma}(\lambda)=\rho(H ; 1)+\rho(\bar{H} ; 1)
$$

was introduced for a smooth Hamiltonian path $\lambda=\phi_{H}$ in Oh5, Oh6]. In Oh5, Oh6], we denoted by $\gamma(H)$ but we change its notation here by leng $(\lambda)$ not to confuse it with the same notation used for the spectral norm function $\gamma: \operatorname{Ham}(M, \omega) \rightarrow$ $\mathbb{R}$ below. leng ${ }_{\gamma}$ was proven to be non-negative and to depend only on the pathhomotopy class of $\lambda=\phi_{H}$ in $\operatorname{Ham}(M, \omega)$.

Definition 5.4 (Spectral length). Let $\lambda \in \mathcal{P}^{\text {ham }}(\operatorname{Symp}(M, \omega), i d)$ and $H$ be a Hamiltonian such that $\lambda=\phi_{H}$. Then we define the function

$$
\operatorname{leng}_{\gamma}: \mathcal{P}^{\text {ham }}(\operatorname{Symp}(M, \omega), i d) \rightarrow \mathbb{R}_{+}
$$

by setting $\operatorname{leng}_{\gamma}(\lambda)=\gamma(H)$. We call $\operatorname{leng}_{\gamma}(\lambda)$ the spectral length of $\lambda$.

Again the uniqueness of continuous Hamiltonians enables us to extend the definition to the continuous Hamiltonian paths.

Proposition 5.5. The spectral length function $\gamma$ extends to a continuous function

$$
\operatorname{leng}_{\bar{\gamma}}: \mathcal{P}_{\infty}^{\text {ham }}(\operatorname{Sympeo}(M, \omega), i d) \rightarrow \mathbb{R}
$$

with the definition

$$
\operatorname{leng}_{\bar{\gamma}}(\lambda)=\lim _{i \rightarrow \infty} \operatorname{leng}_{\gamma}\left(\phi_{H_{i}}\right)
$$

for a (and so any) sequence $\left(\phi_{i}, H_{i}\right) \rightarrow \lambda$ in $C^{0}$-Hamiltonian topology.

Proof. The proof is similar to that of the Hofer length leng in that it is based on the uniqueness of Hamiltonians and the triangle inequality

$$
\operatorname{leng}_{\gamma}(\lambda \mu) \leq \operatorname{leng}_{\gamma}(\lambda)+\operatorname{leng}_{\gamma}(\mu)
$$

and so omitted.

Recall that for a smooth Hamiltonian $H$ each $\rho\left(\phi_{H} ; a\right)=\rho(H ; a)$ is associated to a periodic orbit of Hamilton's equation $\dot{x}=X_{H}(t, x)$ and corresponds to the action of the periodic orbit, at least for the rational symplectic manifold. (See [Oh5, Oh7].) In this regard, the following question seems to be of fundamental importance.

Question 5.6. What is the meaning of the extended spectral length $\operatorname{leng}_{\bar{\gamma}}(\lambda)$ in regard to the dynamics of continuous Hamiltonian flows?

In Oh6, the author has introduced the notion of spectral displacement energy. The following is the analog of the definition from [Oh6] of the spectral displacement energy in the continuous Hamiltonian category. 
Definition 5.7 (Spectral displacement energy). Let $A \subset M$ be a compact subset. We define the spectral displacement energy, denoted by $e_{\bar{\gamma}}(A)$, of $A$ by

$$
e_{\bar{\gamma}}(A)=\inf _{\lambda}\left\{\operatorname{leng}_{\bar{\gamma}}(\lambda) \mid A \cap \lambda(1)(A)=\emptyset, \lambda \in \mathcal{P}_{\infty}^{\text {ham }}(\operatorname{Sympeo}(M, \omega), i d)\right\} .
$$

By unravelling the definitions of Hamiltonian homeomorphisms and of the spectral displacement energy, we also have the following theorem whose proof will be the same as the Hofer displacement energy case and so omitted.

Theorem 5.8. We have $e_{\bar{\gamma}}(A)=e_{\gamma}(A)$ for any compact subset $A \subset M$.

Again based on this theorem, we just denote the spectral displacement energy of $A$ even in the continuous Hamiltonian category by $e_{\gamma}(A)$. Then we have the following theorem

Theorem 5.9. For every $\psi \in \operatorname{Sympeo}(M, \omega)$ we have

$$
e_{\gamma}(A)=e_{\gamma}(\psi(A)) \text {. }
$$

Proof. We note that $h(A) \cap A=\emptyset$ if and only if $\psi h \psi^{-1}(\psi(A)) \cap \psi(A)=\emptyset$. Furthermore $h \in \operatorname{Hameo}(M, \omega)$ if and only if $\psi h \psi^{-1} \in \operatorname{Hameo}(M, \omega)$. This finishes the proof.

Next we recall that in $\mathrm{Oh} 5$, we introduced the non-negative function

$$
\gamma(\phi):=\inf _{e v_{1}(\lambda)=\phi} \operatorname{leng}_{\gamma}(\lambda)=\inf _{H \mapsto \phi}\{\rho(H ; 1)+\rho(\bar{H} ; 1)\} .
$$

and proved that it satisfies the properties of a bi-invariant norm on $\operatorname{Ham}(M, \omega)$ which we called the spectral norm. The following definition extends this definition to $\operatorname{Hameo}(M, \omega)$.

Definition 5.10 (Spectral norm). Let $h \in \operatorname{Hameo}(M, \omega)$ and consider continuous Hamiltonian paths $\lambda \in \mathcal{P}_{\infty}^{\text {ham }}(\operatorname{Sympeo}(M, \omega), i d)$ with $\overline{e v}_{1}(0)=h$. We denote by $\lambda \mapsto h$ if $\overline{e v}_{1}(\lambda)=h$. We define $\bar{\gamma}$ by

$$
\bar{\gamma}(h)=\inf _{\lambda}\left\{\operatorname{leng}_{\bar{\gamma}}(\lambda) \mid \lambda \in \mathcal{P}_{\infty}^{\text {ham }}(\operatorname{Sympeo}(M, \omega), i d), \overline{e v}_{1}(\lambda)=h\right\} .
$$

The following establishes the analogs to all the properties of invariant norm in this continuous Hamiltonian context.

Theorem 5.11. The generalized spectral function $\bar{\gamma}: \operatorname{Hameo}(M, \omega) \rightarrow \mathbb{R}_{+}$satisfies all the properties of an invariant norm stated in Theorem 4.8

Proof. The proof will be essentially the same as that of the Hofer norm once the following continuity lemma for the smooth case is proved.

Lemma 5.12. The function $\gamma: \operatorname{Ham}(M, \omega) \rightarrow \mathbb{R}_{+}$is continuous in the Hamiltonian topology of $\operatorname{Ham}(M, \omega)$.

Proof. Let $H \mapsto \phi$ and $K \mapsto \psi$. Then the triangle inequality of $\gamma$ and the inequality $\gamma(\phi) \leq\|\phi\|$ imply

$$
|\gamma(\phi)-\gamma(\psi)| \leq \gamma\left(\phi^{-1} \psi\right) \leq\left\|\phi^{-1} \psi\right\| \leq\|\bar{H} \# K\| .
$$

In particular, we have

$$
|\gamma(\phi)-\gamma(\psi)| \leq \inf _{H \mapsto \phi, K \mapsto \psi}\|\bar{H} \# K\| .
$$


Now let $\phi \in \operatorname{Ham}(M, \omega)$ and $\varepsilon>0$ be given. Recalling the fact that $e v_{1}$ is an open map (see Corollary $3.17[\mathrm{OM}])$ we consider the open neighborhood $e v_{1}\left(\mathcal{U}\left(\phi_{H}, \varepsilon_{1}, \varepsilon_{2}\right)\right)$ of $\phi$ where $\phi_{H}^{1}=\phi$. Now let $\psi \in e v_{1}\left(\mathcal{U}\left(\phi_{H}, \varepsilon_{1}, \varepsilon_{2}\right)\right)$ i.e., $\psi=\phi_{K}^{1}$ for some $\phi_{K} \in \mathcal{U}\left(\phi_{H}, \varepsilon_{1}, \varepsilon_{2}\right)$. Then we have

$$
\|\bar{H} \# K\|_{\infty} \leq \varepsilon_{1}, \quad \bar{d}\left(\phi_{H}, \phi_{K}\right) \leq \varepsilon_{2}
$$

by the definition of $\mathcal{U}\left(\phi_{H}, \varepsilon_{1}, \varepsilon_{2}\right)$. Therefore if we choose $\varepsilon_{1}=\varepsilon$ and $\varepsilon_{2}$ is any finite number, we have $|\gamma(\phi)-\gamma(\psi)|<\varepsilon$ which proves the continuity of $\gamma$ in the strong Hamiltonian topology.

We omit the rest of the details of the proof referring to the corresponding proofs of Theorem 4.8 .

Question 5.13. The following questions seem to be interesting to study.

(1) Is $\gamma($ or $\bar{\gamma})$ continuous in the $C^{0}$-topology?

(2) Does the following identity

$$
\left.\bar{\gamma}\right|_{\operatorname{Ham}(M, \omega)}=\gamma
$$

hold? This is the spectral analog to Müller's question, Question 4.9 .

\section{CAlabi QUASI-MORPhism ON $\mathcal{P}_{\infty}^{\text {ham }}\left(\operatorname{Sympeo}\left(S^{2}\right), i d\right)$}

In the rest of this section, we will restrict to the case of the sphere $S^{2}$ with the standard symplectic form $\omega_{S^{2}}$ on it. Omitting the symplectic form $\omega_{S^{2}}$ from their notations, we just denote by $\mathcal{P}_{\infty}^{\text {ham }}\left(\operatorname{Sympeo}\left(S^{2}\right), i d\right)$, $\operatorname{Hameo}\left(S^{2}\right)$ the groups of continuous Hamiltonian paths and of Hamiltonian homeomorphisms on $S^{2}$ respectively, and so on.

We first state the following proposition which is the path space version of Theorem $3.1[\mathrm{EP}]$ by Entov and Polterovich.

Proposition 6.1. Consider $S^{2}$ with the standard symplectic form $\omega_{S^{2}}$ on it. Let $H, F$ be smooth normalized Hamiltonians satisfying. Then the spectral invariant $\rho(H ; 1)$ satisfies

$$
\left|\rho\left(\phi_{H} \phi_{F} ; 1\right)-\left(\rho\left(\phi_{H} ; 1\right)+\rho\left(\phi_{F} ; 1\right)\right)\right| \leq R
$$

for some constant $R=R\left(S^{2}\right)>0$ depending only on $\omega_{S^{2}}$ but independent of $H, F$. In particular, the map

$$
\rho(\cdot ; 1): \mathcal{P}^{\text {ham }}\left(\operatorname{Symp}\left(S^{2}\right), i d\right) \rightarrow \mathbb{R}
$$

defines a quasi-morphism.

Proof. The inequality

$$
\rho\left(\phi_{H} \phi_{F} ; 1\right)-\left(\rho\left(\phi_{H} ; 1\right)+\rho\left(\phi_{F} ; 1\right)\right) \leq 0
$$

is nothing but a special case of the triangle inequality (6.2). The existence of a constant $R>0$ such that

$$
\rho\left(\phi_{H} \phi_{F} ; 1\right)-\left(\rho\left(\phi_{H} ; 1\right)+\rho\left(\phi_{F} ; 1\right)\right) \geq-R
$$

was proved by Entov and Polterovich (See the proof of Theorem $3.1[\mathrm{EP}$ ] in the context of the covering space $\widetilde{\operatorname{Ham}}\left(S^{2}\right)$ but its proof equally applies to the context of the path space). Combination of (6.2) and (6.3) finishes the proof. 
We refer to $\mathrm{GG}$, [EP for the general discussion on the basic properties of the quasi-morphism.

Based on this quasi-morphism $\rho(\cdot ; 1)$, Entov and Polterovich defined a homogeneous quasi-morphism on the universal covering space $\widetilde{\operatorname{Ham}}\left(S^{2}, \Omega\right)$

$$
\widetilde{\mu}: \widetilde{\operatorname{Ham}}\left(S^{2}, \Omega\right) \rightarrow \mathbb{R}
$$

by the formula

$$
\widetilde{\mu}(\widetilde{\phi})=\left(\int_{S^{2}} \omega_{S^{2}}\right) \cdot \lim _{i \rightarrow \infty} \frac{\rho\left(\widetilde{\phi}^{m} ; 1\right)}{m} .
$$

We like to point out that due to the different conventions used in [EP], the negative sign in the equation (17) EP does not appear in our definition.

Obviously this definition of homogeneous quasi-morphism can be lifted to the level of Hamiltonian paths :

Definition 6.2. We define a homogeneous quasimorphism

$$
\mu^{\text {path }}: \mathcal{P}^{\text {ham }}\left(\operatorname{Symp}\left(S^{2}\right), i d\right) \rightarrow \mathbb{R}
$$

by the same formula

$$
\mu^{\text {path }}(\lambda)=\left(\int_{S^{2}} \omega_{S^{2}}\right) \cdot \lim _{i \rightarrow \infty} \frac{\rho\left(\lambda^{m} ; 1\right)}{m} .
$$

From the definition above and the hamiltonian-continuity of $\rho(\cdot ; 1)$, it follows that $\mu^{\text {path }}$ is also hamiltonian-continuous.

The following two propositions concerning the quasi-morphism $\mu^{\text {path }}$ were essentially proved by Entov and Polterovich EP.

Proposition 6.3 (Compare with Proposition $3.3[\mathrm{EP}]$ ). Suppose that $U \subset S^{2}$ that is displaceable, i.e., there exists $\phi \in \operatorname{Ham}\left(S^{2}\right)$ such that $\phi(\bar{U}) \cap \bar{U}=\emptyset$. Then we have the identity

$$
\mu^{\text {path }}(\lambda)=\mathrm{Cal}^{\text {path }}(\lambda)
$$

for all $\lambda$ with

$$
\operatorname{supp} \lambda \subset U \text {. }
$$

Entov and Polterovich called this property the Calabi property of a quasi-morphism. We recall that $\operatorname{Cal}(\lambda)$ is defined by the integral

$$
\mathrm{Cal}^{\text {path }}(\lambda)=\int_{0}^{1} \int_{M} H(t, x) \Omega_{\omega}
$$

when $\lambda=\phi_{H}$. Here $\Omega_{\omega}$ is the Liouville volume form normalized so that $\int_{M} \Omega_{\omega}=1$.

Proposition 6.4 (Proposition $3.4[\mathrm{EP}]$ ). The quasi-morphism $\mu^{\text {path }}$ pushes down to a homogeneous quasi-morphism $\mu: \operatorname{Ham}\left(S^{2}\right) \rightarrow \mathbb{R}$. Furthermore $\mu$ is continuous on $\operatorname{Ham}\left(S^{2}\right)$ with respect to the Hamiltonian topology defined in Definition 3.7 OM].

Proof. The proof of the first fact verbatim follows from that of Proposition 3.4 [EP]. The continuity statement immediately follows from the hamiltonian-continuity of $\rho(\cdot ; 1)$ and the definition of the Hamiltonian topology on $\operatorname{Ham}\left(S^{2}\right)$ in Definition $3.7 \mathrm{OM}$. 
For any given open subset $U \subset S^{2}$, we denote by

$$
\mathcal{P}^{\text {ham }}\left(\operatorname{Symp}_{U}\left(S^{2}\right), i d\right)
$$

the set of Hamiltonian paths supported in $U$.

An immediate corollary of these two propositions is the following homomorphism property of $\mu$ restricted to $\mathcal{P}^{\text {ham }}\left(\operatorname{Symp}_{U}\left(S^{2}\right), i d\right)$.

Corollary 6.5. Suppose that $U$ is an open subset of $S^{2}$ such that $\bar{U}$ is displaceable on $S^{2}$ and let

$$
\lambda_{1}, \lambda_{2} \in \mathcal{P}^{\text {ham }}\left(\operatorname{Symp}_{U}\left(S^{2}\right), i d\right) .
$$

Then we have

$$
\mu^{\text {path }}\left(\lambda_{1} \lambda_{2}\right)=\mu^{\text {path }}\left(\lambda_{1}\right)+\mu^{\text {path }}\left(\lambda_{2}\right) .
$$

Now we extend all the above discussions to the level of topological Hamiltonian paths. But these generalization immediately follow once we know the facts that

(1) $\rho(\cdot ; 1)$ has been extended to $\mathcal{P}_{\infty}^{\text {ham }}(\operatorname{Sympeo}(M, \omega), i d)$ for an arbitrary closed symplectic manifold, i.e., in particular for $\left(S^{2}, \omega_{S^{2}}\right)$ in section 5 .

(2) In addition, this extension is hamiltonian-continuous, i.e, continuous in the Hamiltonian topology.

We summarize the above discussion into the following theorem.

Theorem 6.6. We have an extension of $\mu^{\text {path }}: \mathcal{P}^{\text {ham }}\left(\operatorname{Symp}\left(S^{2}\right), i d\right) \rightarrow \mathbb{R}$ to a quasi-morphism

$$
\bar{\mu}^{\text {path }}: \mathcal{P}_{\infty}^{\text {ham }}\left(\operatorname{Sympeo}\left(S^{2}\right), i d\right) \rightarrow \mathbb{R}
$$

that satisfies all the analogs to Proposition 6.4 and the Calabi property.

Now we state the following conjecture, which we believe will play an important role in the study of simpleness question of the area preserving group of $S^{2}$ (and also of $D^{2}$ ). Recall from $\mathrm{EP}$ that the corresponding fact was proved by Entov and Polterovich for the group $\operatorname{Ham}\left(S^{2}\right)$ of smooth Hamiltonian diffeomorphisms on $S^{2}$.

Conjecture 6.7. Let $\bar{\mu}^{\text {path }}: \mathcal{P}_{\infty}^{\text {ham }}\left(\operatorname{Sympeo}\left(S^{2}\right), i d\right) \rightarrow \mathbb{R}$ be the above extension of the homogeneous Calabi quasi-morphism given in (6.5). This pushes down to a homogeneous quasi-morphism $\bar{\mu}: \operatorname{Hameo}\left(S^{2}\right) \rightarrow \mathbb{R}$ that satisfies

$$
\bar{\mu}^{\text {path }}=\bar{\mu} \circ \overline{e v}_{1} .
$$

In particular, $\bar{\mu}^{\text {path }}(\lambda)$ depends only on the time-one map $\lambda(1)$ of $\lambda$ as long as $\lambda$ lies in $\mathcal{P}_{\infty}^{\text {ham }}\left(\right.$ Sympeo $\left.\left(S^{2}\right), i d\right)$. Furthermore it satisfies

$$
\bar{\mu}(\phi)=\overline{\mathrm{Cal}}_{D^{+}}(\phi)
$$

for any $\phi \in \operatorname{Hameo}\left(S^{2}\right)$ as long as $\lambda(1)=\phi$ for a continuous Hamiltonian path $\lambda$ supported in $\operatorname{Int}\left(D^{+}\right)$.

An immediate corollary of Conjecture 6.7 and of the Calabi property of $\bar{\mu}^{\text {path }}$ would be the solution to the following conjecture

Conjecture 6.8. The Calabi homomorphism Cal : $\operatorname{Ham}\left(D^{2}, \partial D^{2}\right) \rightarrow \mathbb{R}$ is extended to a homomorphism

$$
\overline{\mathrm{Cal}}: \operatorname{Hameo}\left(D^{2}, \partial D^{2}\right) \rightarrow \mathbb{R}
$$

that is continuous in Hamiltonian topology. 
In the next section, we will explain how validity of this conjecture together with the smoothing theorem $\mathrm{Oh} 8$, would imply properness of $\operatorname{Hameo}\left(D^{2}, \partial D^{2}\right)$ in of $H_{o m e o}\left(D^{2}, \partial D^{2}\right)$ and hence lead to a proof of non-simpleness of $H_{o m e o}\left(D^{2}, \partial D^{2}\right)$.

\section{Discussion : A Wild AREA PRESERVing homeOMORPHiSM on $D^{2}$}

In this section, we will describe an example of a compactly supported area preserving homeomorphism in $\operatorname{Sympeo}\left(D^{2}, \partial D^{2}\right)$ that would not be contained in Hameo $\left(D^{2}, \partial D^{2}\right)$, if Conjecture 6.8 should hold. Then this would imply that Hameo $\left(D^{2}, \partial D^{2}\right)$ is a proper normal subgroup of $\operatorname{Sympeo}\left(D^{2}, \partial D^{2}\right)$, by Theorem 2.3. Combination the above chain of statements would give rise to non-simpleness of $\operatorname{Homeo}^{\Omega}\left(D^{2}, \partial D^{2}\right)$, via the following theorem which is a corollary of the smoothing theorem from $\mathrm{Oh} 8$.

Theorem 7.1 (Theorem I [Oh8]). We have

$$
\operatorname{Sympeo}\left(D^{2}, \partial D^{2}\right)=\operatorname{Homeo}^{\Omega}\left(D^{2}, \partial D^{2}\right) .
$$

for the standard area form $\Omega$ on $D^{2}$ regarding it also as the symplectic form $\omega=\Omega$.

This being said, we will focus on construction of an example of a wild areapreserving homeomorphism on $D^{2}$. For this description, we will need to consider the conjugate action of rescaling maps of $D^{2}$

$$
R_{a}: D^{2}(1) \rightarrow D^{2}(a) \subset D^{2}(1)
$$

for $0<a<1$ on Hameo $\left(D^{2}, \partial D^{2}\right)$, where $D^{2}(a)$ is the disc of radius $a$ with its center at the origin. We note that $R_{a}$ is a conformally symplectic map and so its conjugate action maps a symplectic map to a symplectic map whenever it is defined.

Furthermore the right composition by $R_{a}$ defines a map

$$
\phi \mapsto \phi \circ R_{a} ; \operatorname{Hameo}\left(D^{2}, \partial D^{2}\right) \rightarrow \operatorname{Hameo}\left(D^{2}(a), \partial D^{2}(a)\right) \subset \operatorname{Hameo}\left(D^{2}, \partial D^{2}\right)
$$

and the composition by $R_{a}^{-1}$ defines a map

$$
\operatorname{Hameo}\left(D^{2}(a), \partial D^{2}(a)\right) \rightarrow \operatorname{Hameo}\left(D^{2}, \partial D^{2}\right) .
$$

We have the following important formula for the behavior of Calabi invariants under the Alexander isotopy.

Lemma 7.2. Let $\lambda$ be a given continuous Hamiltonian path on $D^{2}$ and $\lambda_{a}$ be the map defined by

$$
\lambda_{a}(t, x)= \begin{cases}a \lambda\left(t, \frac{x}{a}\right) & \text { for }|x| \leq a(1-\eta) \\ x & \text { otherwise }\end{cases}
$$

for $0<a \leq 1$. Then $\lambda_{a}$ is also a continuous Hamiltonian path on $D^{2}$ and satisfies

$$
\overline{\mathrm{Cal}}^{\text {path }}\left(\lambda_{a}\right)=a^{4}\left|\overline{\mathrm{Cal}}^{\text {path }}(\lambda)\right| \text {. }
$$

Proof. A straightforward calculation proves that $\lambda_{a}$ is generated by the (unique) continuous Hamiltonian defined by

$$
\operatorname{Dev}\left(\lambda_{a}\right)(t, x)= \begin{cases}a^{2} H\left(t, \frac{x}{a}\right) & \text { for }|x| \leq a(1-\eta) \\ 0 & \text { otherwise }\end{cases}
$$


where $H=\operatorname{Dev}(\lambda)$ : Obviously the right hand side function is the hamiltonian-limit of $\operatorname{Dev}\left(\lambda_{i, a}\right)$ for a sequence $\lambda_{i}$ of smooth hamiltonian approximation of $\lambda$ where $\lambda_{i, a}$ is defined by the same formula for $\lambda_{i}$.

From this, we derive the formula

$$
\begin{aligned}
\overline{\mathrm{Cal}}^{\text {path }}\left(\lambda_{a}\right) & =\int_{0}^{1} \int_{D^{2}(a(1-\eta))} a^{2} H\left(t, \frac{x}{a}\right) \Omega \wedge d t \\
& =a^{4} \int_{0}^{1} \int_{D^{2}} H(t, y) \Omega \wedge d t=a^{4}\left|\overline{\mathrm{Cal}}^{\text {path }}(\lambda)\right|
\end{aligned}
$$

This proves (17.1).

Here comes a construction of an example of wild area preserving homeomorphisms, which is an enhancement of the one described in Example 4.2 [OM].

Example 7.3. With the above preparations, we consider the set of dyadic numbers $\frac{1}{2^{k}}$ for $k=0, \cdots$. Let $(r, \theta)$ be polar coordinates on $D^{2}$. Then the standard area form is given by

$$
\omega=r d r \wedge d \theta .
$$

Consider maps $\phi_{k}: D^{2} \rightarrow D^{2}$ of the form given by

$$
\phi_{k}=\phi_{\rho_{k}}:(r, \theta) \rightarrow\left(r, \theta+\rho_{k}(r)\right)
$$

where $\rho_{k}:(0,1] \rightarrow[0, \infty)$ is a smooth function supported in $(0,1)$. It follows $\phi_{\rho_{k}}$ is an area preserving map generated by an autonomous Hamiltonian given by

$$
F_{\phi_{k}}(r, \theta)=-\int_{1}^{r} s \rho_{k}(s) d s .
$$

Therefore its Calabi invariant becomes

$$
\operatorname{Cal}\left(\phi_{k}\right)=-\int_{D^{2}}\left(\int_{1}^{r} s \rho_{k}(s) d s\right) r d r d \theta=2 \pi \int_{0}^{1} r^{2} \rho_{k}(r) d t .
$$

We now choose $\rho_{k}$ in the following way :

(1) $\rho_{k}$ has support in $\frac{1}{2^{k}}<r<\frac{1}{2^{k-1}}$

(2) For each $k=1, \cdots$, we have

$$
\rho_{k}(r)=2^{4} \rho_{k-1}(2 r)
$$

for $r \in\left(\frac{1}{2^{k}}, \frac{1}{2^{k-1}}\right)$.

(3) $\operatorname{Cal}\left(\phi_{1}\right)=1$.

Since $\phi_{k}$ 's have disjoint supports by construction, we can freely compose without concerning about the order of compositions. It follows that the infinite product

$$
\Pi_{k=0}^{\infty} \phi_{k}
$$

is well-defined and defines a continuous map that is smooth except at the origin at which $\phi_{\rho}$ is continuous but not differentiable: This infinite product can also be written as the homeomorphism having its values given by $\phi_{\rho}(0)=0$ and

$$
\phi_{\rho}(r, \theta)=(r, \theta+\rho(r))
$$

where the smooth function $\rho:(0,1] \rightarrow \mathbb{R}$ is defined by

$$
\rho(r)=\rho_{k}(r) \quad \text { for }\left[\frac{1}{2^{k}}, \frac{1}{2^{k-1}}\right], k=1,2, \cdots .
$$


It is easy to check that $\phi_{\rho}$ is smooth $D^{2} \backslash\{0\}$ and is a continuous map, even at 0 , which coincides with the above infinite product. Obviously the map $\phi_{-\rho}$ is the inverse of $\phi_{\rho}$ which shows that it is a homeomorphism. Furthermore we have

$$
\phi_{\rho}^{*}(r d r \wedge d \theta)=r d r \wedge d \theta \quad \text { on } D^{2} \backslash\{0\}
$$

which implies that $\phi_{\rho}$ is indeed area preserving.

The following lemma will play an important role in our proof of Theorem 7.6.

Lemma 7.4. Let $\phi_{k}$ the diffeomorphisms given in Example 7.3. We have the identity

$$
R_{\frac{1}{2}} \circ \phi_{k-1}^{2^{4}} \circ R_{\frac{1}{2}}^{-1}=\phi_{k}
$$

In particular, we have

$$
\operatorname{Cal}\left(\phi_{k}\right)=\operatorname{Cal}\left(\phi_{k-1}\right)
$$

Proof. Using (17.3), we compute

$$
R_{\frac{1}{2}} \circ \phi_{k-1} \circ R_{\frac{1}{2}}^{-1}(r, \theta)=\left(r, \theta+\rho_{k-1}(2 r)\right)=\left(r, \theta+\frac{1}{2^{4}} \rho_{k}(r)\right)
$$

where the second identity follows from (17.3). Iterating this identity $2^{4}$ times, we obtain (7.4) from (7.3). The equality (7.5) follows from this and (7.1).

An immediate corollary of this lemma and (17.3) is the following

Corollary 7.5. We have

$$
\operatorname{Cal}\left(\phi_{k}\right)=1
$$

for all $k=1, \cdots$

Now we are ready to give the proof of the following theorem.

Theorem 7.6. Validity of Conjecture 6.8 implies that $\phi_{\rho}$ cannot be contained in $\operatorname{Hameo}\left(D^{2}, \partial D^{2}\right)$.

Proof. Suppose to the contrary that $\phi_{\rho} \in \operatorname{Hameo}\left(D^{2}, \partial D^{2}\right)$. Then its Calabi invariant has a finite value which we denote

$$
\overline{\mathrm{Cal}}\left(\phi_{\rho}\right)=C_{1}
$$

for some $C_{1} \in \mathbb{R}$.

Writing $\phi_{\rho}=\psi_{N} \widetilde{\psi}_{N}$ where

$$
\begin{aligned}
\psi_{N} & =\Pi_{i=1}^{N} \phi_{i} \\
\widetilde{\psi}_{N} & =\Pi_{i=N+1}^{\infty} \phi_{i},
\end{aligned}
$$

we derive

$$
C_{1}=\overline{\mathrm{Cal}}\left(\psi_{N}\right)+\overline{\mathrm{Cal}}\left(\widetilde{\psi}_{N}\right)
$$

from the homomorphism property of $\overline{\mathrm{Cal}}$. Here we note that $\psi_{N}$ is smooth and so obviously lies in Hameo $\left(D^{2}, \partial D^{2}\right)$. Therefore it follows from the group property of $\operatorname{Hameo}\left(D^{2}, \partial D^{2}\right)$ that $\widetilde{\psi}_{N}$ lies in $\operatorname{Hameo}\left(D^{2}, \partial D^{2}\right)$ if $\phi_{\rho}$ does so. We also derive

$$
\overline{\operatorname{Cal}}\left(\psi_{N}\right)=\operatorname{Cal}\left(\psi_{N}\right)=\sum_{i=1}^{N} \operatorname{Cal}\left(\phi_{i}\right)=N
$$


from Corollary 7.5, and hence

$$
\overline{\operatorname{Cal}}\left(\widetilde{\psi}_{N}\right)=C_{1}-N
$$

On the other hand, applying (7.4) iteratively to the infinite product

$$
\widetilde{\psi}_{N}=\prod_{i=N+1}^{\infty} \phi_{i}
$$

we show that $\widetilde{\psi}_{N}$ satisfies the identity

$$
\widetilde{\psi}_{N}(r, \theta)= \begin{cases}R_{\frac{1}{2^{N-1}}} \circ \phi_{\rho}^{2^{4(N-1)}} \circ R_{\frac{1}{2^{N-1}}}^{-1}(r, \theta) & \text { for } 0<r \leq \frac{1}{2^{N-1}} \\ (r, \theta) & \text { for } \frac{1}{2^{N-1}} \leq r \leq 1 .\end{cases}
$$

Note the identity

$$
R_{\frac{1}{2^{N-1}}} \circ \phi_{\rho}^{2^{4(N-1)}} \circ R_{\frac{1}{2^{N-1}}}^{-1}(r, \theta)=\left(R_{\frac{1}{2^{N-1}}} \circ \phi_{\rho} \circ R_{\frac{1}{2^{N-1}}}^{-1}\right)^{2^{4(N-1)}}(r, \theta) .
$$

This, the homomorphism property of $\overline{\mathrm{Cal}}$ and Lemma 7.2 applied for $a=2^{(N-1)}$ give rise to

$$
\begin{aligned}
\overline{\mathrm{Cal}}\left(\widetilde{\psi}_{N}\right) & =2^{4(N-1)} \overline{\operatorname{Cal}}\left(R_{\frac{1}{2^{N-1}}} \circ \phi_{\rho} \circ R_{\frac{1}{2^{N-1}}}^{-1}\right) \\
& =2^{4(N-1)}\left(\frac{1}{2^{(N-1)}}\right)^{4} \overline{\operatorname{Cal}}\left(\phi_{\rho}\right)=\overline{\operatorname{Cal}}\left(\phi_{\rho}\right)=C_{1}
\end{aligned}
$$

It is manifest that (7.8) and (7.10) contradict to each other since $N \neq 0$. This finishes the proof.

\section{REFERENCES}

[B] Banyaga, A. Sur la structure du groupe des difféomorphismses qui préservent une forme symplectique, Comm. Math. Helv. 53 (1978), 174-227.

[C] Calabi, E., On the group of auormorphisms of a symplectic manifold, Problems in Analysis (symposium in honor of Bochner), Princeton University Press (1970), 1- 26.

[El] Eliashberg, Y., A theorem on the structure of wave fronts and applications in symplectic topology, Funct. Anal. and its Appl. 21 (1987), 227-232.

[EP] Entov, M., Polterovich, L., Calabi quasimorphism and quantum homology, Int. Math. Res. Not. no 30 (2003), 1635-1676.

[GG] Gambaudo, J.-M., Ghys, É., Commutators and diffeomorphisms of surfaces, Ergod. Th. \& Dynam. Sys. 24 (2004), 1591-1617.

[Gr1] Gromov, M., Pseudo-holomorphic curves in symplectic manifolds, Invent. Math. 82 (1985), 307-347.

[Gr2] Gromov, M., Metric structures for Riemannian and Non-Riemannian spaces, Progress in Math., vol. 152, Birkäuser, Boston, 1998.

[H] Hofer, H. On the topological properties of symplectic maps, Proc. Royal Soc. Edinburgh 115 (1990), 25-38.

[HZ] Hofer, H. and Zehnder, E. Symplectic Invariants and Hamiltonian Dynamics, Birkhäuser, Advanced Texts, Basel-Boston-Berlin, 1994

[LM] Lalonde, F. and McDuff, D., The geometry of symplectic energy, Ann. Math. 141 (1995), 349-371.

[Mu Müller, S., The group of Hamiltonian homeomorphisms in the $L^{\infty}$-norm, preprint, May 2006.

[Oh1] Oh, Y.-G., Symplectic topology as the geometry of action functional, I, J. Differ. Geom. 46 (1997), 499-577.

[Oh2] Oh, Y.-G., Symplectic topology as the geometry of action functional, II, Commun. Anal. Geom. 7 (1999), 1-55. 
[Oh3] Oh, Y.-G., Chain level Floer theory and Hofer's geometry of the Hamiltonian diffeomorphism group, Asian J. Math. 6 (2002), 579-624 ; Erratum 7 (2003), 447-448.

[Oh4] Oh, Y.-G., Normalization of the Hamiltonian and the action spectrum, J. Korean Math. Soc., 42 (2005), $65-83$.

[Oh5] Oh, Y.-G., Construction of spectral invariants of Hamiltonian paths on closed symplectic manifolds, in "The Breadth of Symplectic and Poisson Geometry", Prog. Math. 232, 525 - 570, Birkhäuser, Boston, 2005.

[Oh6] Oh, Y.-G., Spectral invariants, analysis of the Floer moduli space and geometry of Hamiltonian diffeomorphisms, Duke Math. J. 130 (2005), 199 - 295; math.SG/0403083.

[Oh7] Oh, Y.-G., Floer mini-max theory, the Cerf diagram and spectral invariants, submitted, math.SG/0406449.

[Oh8] Oh, Y.-G., $C^{0}$-coerciveness of Moser's problem and smoothing area preserving homeomorphisms, submitted, Nov 2006.

[Oh9] Oh, Y.-G., Locality of continuous Hamiltonian flows and Lagrangian intersection with conormals, J. of Gokova Geometry and Topology, to appear in the inaugural issue, 2007.

[OM] Oh, Y.-G., Müller, S., The group of Hamiltonian homeomorphisms and $C^{0}$ symplectic topology, J. Symp. Geom. (to appear), math.SG/0402210 v3.

[P] Polterovich, L., The Geometry of Group of Symplectic Diffeomorphism, Birkhaüser, 2001.

[Sc] Schwarz, M., On the action spectrum for closed symplectically aspherical manifolds, Pacific J. Math. 193 (2000), 419-461

[V1] Viterbo, C., Symplectic topology as the geometry of generating functions, Math. Ann. 292 (1992), 685-710.

[V2] Viterbo, C., On the uniqueness of generating Hamiltonian for continuous limits of Hamiltonian flows, Internat. Math. Res. Notices, vol 2006, Article ID 34028, 9 pages ; Erratum, ibid, vol 2006, Article ID 38784, 4 pages; math.SG/0509179 v2, 2005.

Department of Mathematics, University of Wisconsin, Madison, Wi 53706 \& Korea Institute for Advanced Study, 207-43 Cheongryangni-dong Dongdaemun-gu, Seoul 130012, KOREA, OH@MATH.WISC.EDU 